Angelika SCHOBER

\title{
DAS DEUTSCHLANDBILD IN DER FRANZÖSISCHEN PRESSE DER ACHTZIGER JAHRE
}

Seit Abschluss des „Vertrages über die deutsch-französische Zusammenarbeit", der am 22. Januar 1963 von Bundeskanzler Konrad Adenauer und dem Präsidenten der französischen Republik Charles de Gaulle im Pariser Elyséepalast unterzeichnet wurde (Elysée-Vertrag), haben sich die Beziehungen zwischen beiden Länder sehr eng gestaltet und gelten in politischer Hinsicht häufig als beispielhaft. Doch wird dies auch ausreichend in der Presse vermittelt? Diese Frage gilt es im Einzelnen zu klären. Um so mehr als der bedeutende Politologe und Kenner der deutsch-französischen Beziehungen Alfred Grosser anlässlich des „Frankfurter Kulturgipfels“ vom 27. und 28. Oktober 1986 schreibt, die Presse trage eine „Mitschuld“ daran, dass die gute Qualität der Beziehungen zwischen beiden Ländern von der Bevölkerung nur unzureichend gewürdigt werde. ${ }^{1}$ Im Folgenden wird gezeigt, welche Informationen über Deutschland zwei bedeutende französische Wochenzeitungen, L'Express und Le Nouvel Observateur, in den achtziger Jahren geben und welches Bild sie auf diese Weise zeichnen. Es wurden diese beiden Presseorgane ausgewählt, da die Arbeiten eines Forschungsseminars am Deutschen Institut der Universität Paris 3 (Sorbonne Nouvelle) ergaben, dass sie in den 70er Jahren eine herausragende Rolle spielten und zwischen 1975 und 1977 das französische Deutschlandbild wesentlich prägten. ${ }^{2}$ Beide

$1 \quad$ Alfred Grosser, "Das Wenige wahren", Frankfurter Allgemeine Zeitung, 1.10.1986. Auf diesem Gipfel, der das Deutsch-französische Kulturabkommen von 1954 ergänzt, beschlossen Bundeskanzler Helmut Kohl und Staatspräsident François Mitterrand die Beziehungen zwischen beiden Ländern im Kulturbereich zu vertiefen, besonders durch einen gemeinsamen deutsch-französischen Fernesehkanal (ARTE), Maßnahmen zur Förderung der Sprache des Partnerlandes und den Ausbau von gemeinsamen Studiengängen im Rahmen einer Deutsch-französischen Hochschule.

2 Cf. Hans Hörling, „L'image de la République fédérale allemande (RFA) dans la presse hebdomadaire française“, in: L'Allemagne vue par la presse française. Cahiers de l'Association pour une lecture critique de la presse, Paris, 23.10.1978, S.14-23.

Kairoer Germanistische Studien 19(2010/2011) 
Zeitungen weisen eine Reihe von Gemeinsamkeiten auf, haben aber auch Unterschiede in Bezug auf ihre allgemeine Redaktionspolitik und Schwerpunktsetzung. Sie verstehen sich als Organe der Reflexion und Analyse, arbeiten also, mit den angelsächsischen Termini der Presseanalyse gesprochen, in erster Linie nicht mit news, sondern mit views. L'Express definiert sich als Nachrichtenmagazin nach dem Vorbild des amerikanischen Magazins Time und des deutschen Nachrichtenmagazins Der Spiegel. Nach Beendigung der französischen Kolonialpolitik im Jahre 1964 definierte JeanJacques Servan-Schreiber die Aufgaben seiner Zeitung in diesem Sinne. Daraufhin verließ Jean Daniel die Redaktion von L'Express und gründet seine eigene Zeitung, Le Nouvel Observateur, mit der Absicht, das linksorientierte Engagement beizubehalten. Und er wollte zudem den Lesern Hintergrundinformationen liefern, die andere Presseorgane nicht bieten. Dieser Anspruch wurde beibehalten, wie ein Werbeprospekt aus dem Jahre 1990 zeigt: „Seit fünfundzwanzig Jahren ist Le Nouvel Observateur seiner Zeit voraus“, wirft einen ,anderen“ Blick auf die Ereignisse und versucht „durch scharfsinnige Bearbeitung der Informationen zum Nachdenken anzuregen“. Was L'Express betrifft, so ist Jean-François Revel zufolge die ganze Geschichte dieser Zeitung von der Absicht geprägt, das Leitmotiv von Jean-Jacques Servan-Schreiber in die Tat umzusetzen, der zufolge den Franzosen „die Wahrheit gesagt werden muss“.” Der Unterschied im prinzipiellen Anspruch ist also relativ gering, doch in Bezug auf den Stil ist zu sagen: In L'Express findet man meistens eine eher neutrale Sprache mit dem Ziel größtmöglicher Objektivität, während Le Nouvel Observateur eine

Das Pariser Goethe Institut veranstaltete 1978 - aufgrund des negativen Deutschlandbildes bedingt durch die Ereignisse vom Herbst 1977 (Stammheim) - eine Gesprächsrunde, in welcher deutsche und französische Journalisten eine Antwort auf die Frage suchten „Ist die Presse in den deutsch - französischen Beziehungen ein Faktor der Verständigung oder des Missverständnisses?" Henri Menudier (Hrsg.), La presse et les relations franco- allemandes. Facteur de compréhension ou de mésentente?, Paris, Goethe-Institut, 1978. Siehe auch Henri Menudier, Das Deutschland der Franzosen in den 70er Jahren, Europa Union Verlag, Bonn 1981.

3 L'Express. L'aventure du vrai, Albin Michel 1979, Vorwort von Jean-François Revel, S.14. Ein Werbeslogan von L'Express lautete entsprechend: „L'Express deckt die ganze Wahrheit auf, die hinter den Ereignissen und Menschen verborgen ist.“, S.216. 
besondere Sprachform entwickelte, die Louis Pinto als „fiktiven Konversationsstil Pariser Intellektueller" bezeichnet. ${ }^{4}$

Das Deutschlandbild von L'Express und Le Nouvel Observateur unterschied sich, wie die Ergebnisse des zitierten Forschungsseminars zeigen, in den 70er Jahren stark. L'Express vertrat eine überwiegend positive Haltung und kritisierte die Reaktionen des bundesdeutschen Staates auf die Herausforderungen durch die „Rote Armee Fraktion“, schwächer als andere französische Presseorgane. Das Hauptinteresse galt der Wirtschaft, gefolgt von Innenpolitik und nationalsozialistischer Vergangenheit; Außenpolitik interessierte wenig. Le Nouvel Observateur beurteilte hingegen die Bundesrepublik sehr kritisch und gab der Befürchtung Ausdruck, sie könne sich in einen totalitären Staat verwandeln. Die Kritik an der Innenpolitik prägte das Gesamtbild und die nationalsozialistische Vergangenheit spielte eine wichtige Rolle. Trotz der prinzipiell skeptischen Einstellung gegenüber Deutschland war Le Nouvel Observateur aber um Meinungsvielfalt bemüht und ließ auch weniger kritische Kommentatoren zu Wort kommen, vor allem Alfred Grosser und Günter Grass. Wir stellten uns deshalb die Frage: Treffen die für die 70er Jahre festgestellten Unterschiede in Schwerpunktsetzung und Beurteilung des Nachbarlandes auch für die achtziger Jahre noch zu?

Zur Beantwortung dieser Frage untersuchten wir im Detail alle Ausgaben beider Zeitungen zwischen dem zwanzigsten und fünfundzwanzigsten Jahrestag des Elysée-Vertrages (1983-1988). Die Auswertung aller fünfhundertzwanzig Ausgaben von L'Express und Le Nouvel Observateur im Untersuchungszeitraum ergab zunächst, dass insgesamt dreihundertvierundneunzig Artikel mit Bezug zu Deutschland erschienen, davon zweihundertvierundzwanzig in L'Express und hundertsiebzig in $L e$

Louis Pinto, L'intelligence en marche : Le Nouvel Observateur, Editions Métaili, Paris 1984, S.25. Beide Zeitungn verwenden manchmal deutsche Wörter in Artikelüberschriften, zum Beispiel: „Ich liebe dich, moi non plus“ (L'Express, 15.1.1983), „RFA: le Kriegsspiel des métallos“" (L'Express, 29.5.1984) oder „Luther mit uns“ (Le Nouvel Observateur, 18.11.1983), eine Anspielung auf den Satz „Gott mit uns“, die im 1.Weltkrieg auf deutschen Gewehren stand. 
Nouvel Observateur. Sie wurden analysiert in Hinblick auf die Fragen: Was erfährt der Leser über Deutschland und die deutsch-französischen Beziehungen? Welche Informationen erhält er über die verschiedenen Bereiche der Politik? Über Wirtschaft, Gesellschaft und Kultur? Welchen Stellenwert hat die Geschichte, besonders die nationalsozialistische Vergangenheit ? Wie viele Artikel beschäftigen sich jedes Jahr mit dem Nachbarland? Auf welchen Themen liegt der Schwerpunkt? Die Artikel wurden hierzu in sieben Rubriken eingeteilt: Innenpolitik einschließlich deutsch-deutsche Beziehungen, deutsch-französische Beziehungen, Außenpolitik, Wirtschaft, Gesellschaft, Kultur, Geschichte, wobei es manchmal Überschneidungen gibt. Die Rubrik Innenpolitik enthält auch Artikel über innenpolitische Auswirkungen internationaler Abkommen sowie Kommentare zur deutschen Identität. Die deutsch-französischen Beziehungen erscheinen im Rahmen des europäischen Einigungsprozesses. Die Detailanalyse der Jahre 1983 bis 1987 wird ergänzt durch einen Vergleich der Dossiers die beide Zeitungen zum fünfundzwanzigsten Jahrestag des ElyséeVertrages im Januar 1988 veröffentlichten. Eine zusammenfassende Auswertung beschließt die Arbeit, die eine Kombination von Analyse und Dokumentation darstellt und in komprimierter Form das vielschichtige Deutschlandbild beider Presseorgane nachzeichnet. ${ }^{5}$ Wir verzichteten auf eine thematische Gliederung des Materials und wählten stattdessen eine chronologische Darstellung, die auf Vollständigkeit Anspruch erhebt. Denn auf diese Weise kann am besten gezeigt werden, welche Informationen tatsächlich den Lesern beider Zeitungen vermittelt werden. Und damit geklärt werden, inwieweit L'Express und Le Nouvel Observateur die gute Qualität der deutsch-französischen Beziehungen vermitteln.

1983

Im Jahr 1983 erscheint fast ein Drittel der im gesamten Untersuchungszeitraum veröffentlichten Artikel über Deutschland und die deutsch-französischen Beziehungen, nämlich hundertdreizehn, davon zweiundfünfzig in L'Express und einundsechzig in Le Nouvel Observateur.

5 Die im Text nicht eigens besprochenen Artikel sind gleichwohl in den Fußnoten angeführt. 
Das starke Interesse gilt vor allem den Aktionen der Friedensbewegung gegen die geplante Stationierung amerikanischer Mittelstreckenraketen als Folge des NATO-Doppelbeschlusses vom 12. Dezember 1979. Sie lösen eine Reihe von Befürchtungen in Frankreich aus. Nur in diesem Jahr publiziert Le Nouvel Observateur mehr Artikel über Deutschland als L'Express.

Innenpolitik und innenpolitische Konsequenzen internationaler Abkommen kommentieren zwanzig Artikel in L'Express und fünfundzwanzig in Le Nouvel Observateur. Für Henri de Bresson und Michel Meyer ist die Kritik der Sozialdemokraten an der Stationierung der Pershing II-Raketen Ausdruck einer ,antiwestlichen“ Stimmung6 und Fred Kupfermann hält die hauptsächlich in den protestantischen Ländern Europas aktive Friedensbewegung für eine „Falle ${ }^{6 / 7}$. Die Herbstdemonstrationen mit der „Belagerung des Bundestages“ finden kein Verständnis, ${ }^{8}$ Anne-Marie Le Gloannec und weitere Journalisten bestätigen André Glucksmanns kritische „Psychoanalyse des Pazifismus“"9. Während in L'Express keine Vertreter der Friedensbewegung $\mathrm{zu}$ Wort kommen, gibt Le Nouvel Observateur dem Schriftsteller Günter Grass und der Bundestagsabgeordneten der Grünen Petra Kelly die Möglichkeit sich zu äußern. ${ }^{10}$ Die Zeitung bemüht sich also um Meinungsvielfalt, doch die Kritik an den „Ökopazifisten“ überwiegt. Auch in einem Kommentar des deutschen Journalisten Peter Scholl-Latour, der meint, der politische Irrealismus der Grünen und die Angst vor einer nuklearen

$6 \quad$ Henri de Bresson, "Allemagne: la gauche lâche les fusées", EX, 3.6.1983, S.89; Michel Meyer, "Allemagne: le reniement des sociaux-démocrates", EX, 25.11.1983, S.40.

7 Fred Kupfermann, "Les pièges du pacifisme”, EX, 10.6.1983, S.98-99.

8 Henri de Bresson, "Pacifistes: comment s'en servir", EX, 8.7.1983; Jérôme Dumoulin, "Allemagne fédérale: saison des manifs", EX, 26.8.1983, S.43 44; Michèle Georges, "Pacifistes: le siège du Bundestag", EX, 25.11.1983, S.42.

9 C.d.E. "Les pacifistes s'en vont en guerre", EX, 21.10.1983, S.111; Anne-Marie Le Gloannec, "R.F.A.: qui a peur des Pershing?", EX, S.112-113; Jérôme Dumoulin, "Fusées: le jour J", EX, 25.11.1983, S.34-39; André Glucksmann, "Document: psychanalyse du pacifisme", EX, 11.11.1983, S.134-138. Siehe hierzu auch sein Buch La force du vertige, Grasset et Fasquelle, Paris 1983.

10 Gérard Sandoz, Interview mit Petra Kelly, "Arrêtez-cela", NO, 25.2.1983, S.39; ders. Interview mit Günter Grass, "Allemagne. Pourquoi nous refusons les Pershing”, NO, 14.10.1983, S.53. 
Apokalypse würde den ,großen kollektiven Ängsten der Deutschen in der Vergangenheit" entsprechen. ${ }^{11}$ Tatsächlich steht das Thema Angst $1983 \mathrm{im}$ Mittelpunkt der Deutschlandberichterstattung von Le Nouvel Observateur. Und zwar sowohl die deutsche Angst vor Raketen und Arbeitslosigkeit, als auch jene der Franzosen vor einem deutschen Neutralismus und neuem Nationalismus. ${ }^{12}$ Anlässlich der Ratifizierung des NATO-Doppelbeschlusses am 22. November 1983 analysieren Jean Daniel, Jacques Julliard und Katharina von Bülow die ,wahre Bedrohung“"13. Wobei vor allem die kritische Haltung der Friedensbewegung gegenüber den Vereinigten Staaten irritiert sowie die Wandlung der SPD, die unter Hans Jochen Vogel von dem durch Helmut Schmidt initiierten Doppelbeschluss abrückt und sich den Positionen der Grünen annähert. Ein Artikel über die Ausweisung von Pazifisten aus der DDR unterstreicht, was François Mitterrand am 20. Januar 1983 in seiner Rede vor dem Deutschen Bundestag zur Feier des zwanzigsten Jahrestags des Elysée-Vertrages sagte: „Die Pazifisten sind im Westen, die Raketen im Osten." "14

Weder L'Express noch Le Nouvel Observateur zeigen Sympathie für die Rüstungsgegner, doch es gibt Nuancen in der Bewertung. In L`Express unterstützt Michel Meyer die Politik Helmut Kohls und Jérôme Dumoulin erklärt den Zwiespalt der deutschen Politik: Sie werde hin- und hergerissen

11 Josette Alia, Interview mit Peter Scholl-Latour, "Dissuasion: les Allemands, l'autruche et la bombe", NO, 25.2.1983, S.36.

12 Titelgeschichte: "Spécial Allemagne. De qui ont peur les Allemands", NO, 25.2.1983; Jean Daniel, “Chirac ou Vogel ?”, ebd., S.49.

13 Titelgeschichte: "Pershing: les vraies menaces", NO, 18.11.1983 ; Jean Daniel, ebd., S.32 ; Jaques Julliard, "Eglise: la fusée des évêques", ebd.,S.34 ; Katharina von Bülow, "Pourquoi j'ai peur du pacifisme”, ebd., S.118-121.

14 Gérard Sandoz, "R.D.A.; La paix hors le Mur", NO, 24.6.1983, S.33. Weitere Artikel zu Friedensbewegung und Kritik am Doppelbeschluss: Gérard Sandoz, "Allemagne: Si l'Amérique doit nous lâcher...", NO, 14.1.1983, S.43 ; "Pershing: la sagesse selon Kohl", NO, 5.8.1983, S.32 ; ders., "Pacifisme: la grande offensive d'automne", NO, 26.8.1983, S.38 ; ders., "Allemagne: Schmidt vote Pershing", NO, 30.9.1983, S.72 ; ders., "Allemagne: flirt avec les Pershing", NO, 7.10.1983, S.42; ders., "Allemagne: haro sur l'Amérique", NO, 28.10.1983; ders., "Allemagne: le S.P.D. digère les Verts", NO, 26.11.1983, S.50.S.42 ; Katharina von Bülow, "Berlin: la détente tout prix", $N O$, 29.4.1983, S.51; Jean Daniel, NO, 14.10.1983, S.37; C.F. Jullien, "Episcopat: la conversion de Lourdes", NO, 18.11.1983, S.35. 
zwischen „Staatsräson“ und „nationaler Räson“, da erstere verlangt, ein glaubwürdiger NATO-Partner zu sein, während letztere mit Blick auf den zweiten deutschen Staat der Abrüstung den Vorzug geben möchte. ${ }^{15}$ Le Nouvel Observateur befindet sich in einem Dilemma. Wegen seines linksorientierten Engagements werden, trotz Kritik an der Friedensbewegung, positive Stellungnahmen zugunsten der liberal-konservativen Bundesregierung vermieden. Ähnliches gilt in Bezug auf Franz Josef Strauß. Während L'Express dem bayerischen Ministerpräsidenten darin Recht gibt, dass die Grünen nicht reif für die parlamentarische Demokratie seien, übergeht Le Nouvel Observateur die Kritik von Strauß an den „Ökopazifisten“. Nach der Bundestagswahl vom 6. März 1983 bedauert Gérard Sandoz, dass „,der Bayer immer noch da ist" und weiterhin Günter Grass und Heinrich Böll als Vertreter einer „,entarteten Kultur“ beschimpfen könne. ${ }^{16}$

Die Wahl zum Deutschen Bundestag kommentiert Le Nouvel Observateur in fünf, L'Express in vier Artikeln. Beide Zeitungen informieren über den SPD-Kanzlerkandidaten Hans Jochen Vogel und seine Kritik an der Nachrüstung. ${ }^{17}$ Sie zeichnen zudem ein relativ düsteres Gesamtbild der Bundesrepublik. In Le Nouvel Observateur prognostiziert Gérard Sandoz soziale, politische und wirtschaftliche Spannungen ${ }^{18}$, in L'Express zitiert Jérôme Dumoulin das deutsche Nachrichtenmagazin stern und spricht von

15 Michel Meyer, "Portrait. Helmut Kohl: une force qui va", EX, 7.10.1983, S.168-169; Jérôme Dumoulin, “Allemagne fédérale: raison d'Etat ou raison de nation”, EX, 21.1.1983.

16 Michèle Georges, "Allemagne: la petite flamme verte", EX, 4.2.1983, S.71. Gérard Sandoz, "Les couleurs de la nouvelle droite", NO, 11.3.1983, S.58.

Zum Bild des bayerischen Ministerpräsidenten in beiden Zeitungen siehe auch meinen Aufsatz „Bayern und München in den französischen Wochenzeitungen L'Express und Le Nouvel Observateur 1983-1988“/ „La Bavière et Munich dans les hebdomadaires français L'Express et Le Nouvel Observateur 1983-1988“, in: Pierre Albert, Ursula Koch, Detlef Schröder (Hrsg.), Deutsch-französische Medienbilder. Journalisten und Forscher im Gespräch/ Images médiatiques franco-allemandes. Un dialogue entre journalistes et chercheurs, Verlag Reinhard Fischer, München 1993, S.171-186.

Gérard Sandoz, "Les couleurs de la nouvelle droite", NO, 11.3.1983, S.58.

17 Gérard Sandoz, "Portrait. Un certain Hans Jochen Vogel", NO, 8.1.1983, S.37; Jérôme Dumoulin, "Allemagne: l'option Vogel", EX, 21.1.1983, S. 58-59. 
einer „Welle der Frustration“. Anders als Gérard Sandoz sieht er aber Zeichen für Optimismus: Bundespräsident Karl Carstens erscheint als „Optimist aus Prinzip“, der Bundeskanzler als „Optimist von Natur aus“. Jérôme Dumoulin meint zudem, die ,joviale Haltung“ Helmut Kohls werde auf die Bundesrepublik abfärben. ${ }^{19}$ Nach der Bundestagswahl kommentiert der Frankfurter Soziologe Jürgen Habermas, der sich selbst als „Pazifist der ersten Stunde" versteht, die politische Landschaft. Er befürchtet einen Rechtsruck mit Rücknahme von Reformen der siebziger Jahre, etwa im Scheidungsrecht, sowie Rückschritten in der Umweltpolitik. Zugleich distanziert er sich aber von den Grünen, deren „nationale Strömung“ ihm missfällt. ${ }^{20}$ François Schlosser hält den Wahlsieg des Bundeskanzlers für ambivalent. Wiedergewählt, weil ihm die Mehrheit der Deutschen mehr wirtschaftspolitische Kompetenz zutraut als seinem Herausforderer Hans Jochen Vogel, fehle ihm gleichwohl eine breite Unterstützung durch die Bevölkerung in Hinblick auf die Nachrüstung ${ }^{21}$. Gérard Sandoz meint, Franz Josef Strauß werde mit $67 \%$ der Stimmen für die CSU in Bayern weiterhin großen Einfluss auf die Bonner Politik ausüben ${ }^{22}$. L'Express sieht den Kanzler als absoluten Sieger, der für die nächsten vier Jahre „freie Hand“ hat und Henri de Bresson zufolge wurde Franz Josef Strauß auf Bundesebene „matt“ gesetzt. ${ }^{23}$

Die Kommentierung weiterer innenpolitischer Themen weist einige Unterschiede auf. Le Nouvel Observateur befürchtet eine stärkere Rechtsorientierung der Bundesregierung und spricht von einer ,gefährlichen Wende" angesichts geplanter Verschärfungen des Demonstrationsrechts (,Vermummungsverbot"“). ${ }^{24}$ L'Express sieht keine derartige Gefahr. Der Leser

\footnotetext{
19 Jérôme Dumoulin, "Le choix du président", EX, 7.1.1983, S. 83.

20 Jürgen Habermas, "Ce que les Verts peuvent apporter...", NO, 11.3.1983, S.58.

21 François Schlosser, "Une victoire ambiguë", NO, 11.3.1983, S.60.

22 Gérard Sandoz, "Les couleurs de la nouvelle droite", NO, 11.3.1983, S.58; ders., "R.F.A. : que faire de Strauss?", NO, 18.3.1983, S.66.

23 "Allemagne: Kohl chancelier pour quatre ans: vainqueur et les mains libres", EX, 11.3.1983, S. 92-95 ; Henri de Bresson, "Monde: Strauss: échec et mat", EX, 25.3.1983, S. 94-95. 
erhält vielmehr Informationen über die Kritik vieler Bundesbürger an der Volkszählung sowie über die Flick-Affäre (Parteispenden), in die auch Wirtschaftsminister Otto Graf Lambsdorff verwickelt ist. ${ }^{25}$ Obwohl Le Nouvel Observateur 1983 mehr Artikel zu innenpolitischen Ereignissen veröffentlicht, ist die Bandbreite der behandelten Themen in L'Express größer.

Die deutsch-französischen Beziehungen erfahren etwa gleich viel Beachtung, fünf Artikel erscheinen hierzu in L'Express, sechs in Le Nouvel Observateur. Zum zwanzigsten Jahrestag des Vertrages über die deutschfranzösische Zusammenarbeit unterstreicht in L'Express Jérôme Dumoulin die Bedeutung Deutschlands für die europäische Verteidigung. ${ }^{26}$ Der Soziologe Raymond Aron unterstützt der Sache nach die Rede des französischen Staatspräsidenten François Mitterrand vor dem Deutschen Bundestag am 20.Januar 1983, fragt aber, ob es geschickt war, in die interne Diskussion einzugreifen. ${ }^{27}$ Im weiteren Verlauf des Jahres betont Jérôme Dumoulin die Bedeutung der Bundesrepublik für Frankreich und der Historiker Josef Rovan analysisert, mit welchem Deutschland Frankreich es zu tun hat und haben möchte. Michel Jacques bedauert, dass kurzfristige Überlegungen das Verhältnis zwischen beiden Staaten belasten. ${ }^{28}$ Le Nouvel Observateur spricht sogar von einer „Betonhochzeit“. Das Verhältnis zwischen Bonn und Paris ist François Schlosser zufolge problematisch, neben „Missverständnissen und Zweideutigkeiten“" gebe es sogar „Verdächtigungen“. Hauptprobleme sind die Sicherheitsfrage und, damit verbunden, die Beziehungen der Bundesrepublik zu den Warschauer-Pakt-Staaten. Bonn werde ein immer ,unsicherer“ Partner, die „Grenzen der vor zwanzig Jahren geschlossenen deutsch-französischen Ehe" würden deutlich erscheinen. ${ }^{29}$ Jacques Mornand bedauert den deutschen

\footnotetext{
25 Michèle Georges, "XY 1250 ne répond plus", EX, 15.4.1983, S.94; Michel Meyer, "Allemagne: le scandale Lambsdorff met en péril le gouvernement", EX, 9.12.1983, S.26.

26 Jérôme Dumoulin, "Est-Ouest: l'enjeu allemand", EX, 14.1.1983, S.72-73.

27 Raymond Aron, "Paris et Bonn face aux SS 20", EX, 28.1.1983, S.60.

28 Michel Jacques, "France-R.F.A.: comptes à courte vue", EX, 8.4.1983, S.84-86; Jérôme Dumoulin, "Allemagne: le grand enjeu", EX, 4.3.1983, S.82-85; "Document. Josef Rovan: quelle Allemagne?", EX, 4.3.1983, S.122 ; "Le match France Allemagne" (Statistiken), ebd., 11.3.1983, S.80-81.

François Schlosser, "Paris-Bonn: des noces de béton", NO, 21.1.1983, S.33-35.
} 
Druck auf Frankreich im Währungsbereich. ${ }^{30}$ Dieses eher negative Bild relativiert Jean Daniel jedoch in zwei Artikeln. Er hält die Beziehungen für „herzlich“ und meint, Frankreich und Deutschland säßen ,im gleichen Boot, auch wenn die Bundesrepublik darin komfortabler installiert" sei. ${ }^{31}$ Zum 20. Jahrestag des Elysée-Vertrages unterstreicht der ehemalige Bundeskanzler Willy Brandt die Notwendigkeit guter Beziehungen zwischen Bonn und Paris und eine Umfrage des Deutsch-französischen Jugendwerkes bestätigt das positive Frankreichbild junger Deutscher. ${ }^{32}$ Mitte Mai meint François Schlosser, es werde zwar 1983 ,noch keine Scheidung zwischen Kohl und Mitterrand" geben, doch der Wirtschaftsgipfel von Williamsburg zeigte, dass Deutschland „verstärkt nach anderen Bräuten schielt". Die Schuld an den „verwelkten Blumen“ trage aber allein Bonn, denn die Bundesregierung verfolge, trotz gegenteiliger Beteuerungen und einem scheinbaren Entgegenkommen in Währungsfragen, letztlich nur eigene Exportinteressen. Ein halbes Jahr später hat sich dem Journalisten zufolge die Situation nicht gebessert. Läuft auch „augenscheinlich“ die Ehe gut, sei das Lächeln „nicht ganz ehrlich“. Die Ratifizierung des NATO-Doppelbeschlusses durch den Bundestag könne das französische Misstrauen nicht ausräumen, denn nach wie vor opponiert die Friedensbewegung. ${ }^{33}$

Deutsche Außenpolitik interessiert 1983 beide Wochenzeitungen nur aus der Perspektive der Ost-West-Beziehungen. Sie kommentieren am 8. Juli das Handelsabkommen zwischen der Bundesrepublik Deutschland und der Sowjetunion. ${ }^{34}$ Le Nouvel Observateur hält die Bonner Außenpolitik für zweideutig: Einerseits werden die Wirtschaftsbeziehungen mit der UdSSR ausgebaut, andererseits versucht man, ein verlässlicher Bündnispartner des

\footnotetext{
30 Jacques Mornand, "Le Mark et le Franc", NO, 25.2.1983, S.40.

31 Jean Daniel, "Chirac ou Vogel", NO, 25.2.1983, S.49; ders. "Leur Allemagne", NO, 28.3.1983, S.20

32 "Willy Brandt témoigne", NO, 21.1.1983, S.92; "La France vue par les jeunes Allemands", ebd., S.34.

33 François Schlosser, "Paris-Bonn: les fleurs fanées", NO, 20.5.1983, S.47 ; ders., "MitterrandKohl: la flamme vacille", $N O, 26.11 .1983$, S.53.

34 Henri de Bresson, "Monde. Andropov-Kohl: des menaces bien tempérées", EX, 8.7.1983, S. 54 -55; François Schlosser, "Est-Ouest. Un chancelier culotté", NO, 8.7.1983, S.35.
} 
Westens zu bleiben. L'Express bestätigt diese Analyse. Zu Wirtschaftsfragen erscheint jeweils ein Artikel. L'Express erörtert Gründe der Dynamik der deutschen Wirtschaft und betont, dass anders als in Frankreich auf staatliche Eingriffe verzichtet wird. Le Nouvel Observateur befürchtet einen Sozialabbau zugunsten der Wettbewerbsfähigkeit. ${ }^{35}$ Der Bereich Gesellschaft bleibt weitgehend ausgeblendet, nur L'Express informiert über deutsche Besorgnisse in Bezug auf das Waldsterben. ${ }^{36}$ Zur Kultur veröffentlicht L'Express zwölf, Le Nouvel Observateur vierzehn Artikel. Sie kommentieren weniger das Kulturleben in der Bundesrepublik als deutsche Kultur in Frankreich. Beide Zeitungen kommentieren Peter Schneiders Roman Der Mauerspringer, Le Nouvel Observateur interviewt den Autor. ${ }^{37}$ L'Express thematisiert Michael Grübers Berliner Hamletinszenierung, die Schauspielerin Hanna Schygulla sowie den Modeschöpfer Karl Lagerfeld. ${ }^{38}$ Le Nouvel Observateur zeigt stärkeres Interesse am Ballett. Ralph de Gubernatis berichtet über die Choreographin Pina Bausch anlässlich ihres Pariser Gastspiels und Mathieu Lindon würdigt ihre Leistungen anlässlich des Münchner Theaterfestivals. Die „Fee von Wuppertal“" ist am 12. Dezember sogar Titelbild des Nouvel Observateur. L'Express widmet ihr keinen eigenen Artikel, Simone Dupuis erwähnt sie aber in ihrem Bericht über Rolle der Frauen im internationalen Tanztheater. ${ }^{39}$ Dem hundertsten Todestag von Richard Wagner schenkt Le Nouvel Observateur mehr Beachtung als L'Express. Sechs Journalisten

35 Michel Meyer, "Allemagne: Colbert, connais pas", EX, 18.2.1983, S.65-66 ; Gérard Sandoz, "Allemagne: miracle à la tronçonneuse", NO, 30.12.1983, S.37.

36 Michèle Georges, "La forêt allemande à l'agonie", EX, 20.5.1983, S.105-106.

37 Dominique Fernandez, "Berlin, ou le cœur coupé en deux", EX, 20.5.1983, S.49; Philippe Boyer, Interview mit Peter Schneider, "Lettres allemandes: Berlin, prison dorée", NO, 26.8.1983, S.60.

$38 \quad$ Mathieu Galey, "Berlin: un spectre, des spectres", EX, 21.1.1983, S.13; ders., "Brecht: Las Vegas avant le déluge", EX, 16.12.1983, S.91 ; René Bernard, "Hanna: punk, clown, vamp", EX., 12.5.1983, S.19; Dominique Brabec, "Portrait. Karl Lagerfeld: un provo chez Coco", EX, 11.2.1983, S.19. dames", $E X, 19.8 .1983$, S.8-9. 
schreiben hierzu, L'Express beschränkt sich auf ein Interview mit dem Regisseur Hans Jürgen Syberberg. ${ }^{40}$ Beide Zeitungen erinnern an Frank Kafka anlässlich seines hundertsten Geburtstags. ${ }^{41}$ L'Express bespricht zudem Bücher über Hölderlin und E.T.A. Hoffmann. ${ }^{42}$ Le Nouvel Observateur interessiert sich für das in Lyon aufgeführte antifaschistische Ballett von Kurt Jooss, dem Begründer der Folkwangschule, aus dem Jahre 1937, veröffentlicht eine Stellungnahme von Günter Grass zu Nicaragua und kommentiert die Philosophie Heideggers. ${ }^{43}$ Die Aufarbeitung der Vergangenheit in Werken bekannter deutschsprachiger Autoren wird ebenfalls analysiert. Philippe Boyer zufolge ist „der Buchstabe H wie Hitler“ den Schriftstellern Günter Grass, Peter Härtling, Botho Strauß und Peter Schneider sowie Hans Jürgen Syberberg „wie ein unauslöschliches Siegel“ eingebrannt. ${ }^{44}$ Die gefälschten Hitlertagebücher kommentieren beide Wochenzeitungen, L'Express skizziert in diesem Zusammenhang das Porträt des stern-Herausgebers Henri Nannen. ${ }^{45}$ Geschichte wird aktualisiert anlässlich des fünfhundertsten Geburtstags von Martin Luther. Seine Nachwirkungen auf die deutsche Gesellschaft, besonders die Friedensbewegung, seien nicht $\mathrm{zu}$ übersehen. ${ }^{46}$ Bei diesem

40 Jacques Drillon, Wolfgang Sawaltisch, René Girard, Werner Schroeter, Jean Freusti, Philippe Boyer, "Hommage: pas de requiem pour Wagner", NO, 4.2.1983, S.68-78 ; Hans Jürgen Syberberg, "Wagner n'était pas coupable", EX, 15.4.1983, S.18-23.

41 Ivo Fleischmann/Philippe Boyer, "Centenaire: Kafka pur et simple, Franz Kafka né à Prague", NO, 1.7.1983, S. 6; Jean-Louis Etiène, "Les dernières métamorphoses de Franz Kafka" (Übersetzung des Prozess von Bernard Lortholary, Flammarion), EX, 28.10.1983. Pierre Bertaux/ Dominique Fernandez, "Hölderlin ou le temps d'un poète", EX, 27.5.1983, S.58-59 ; Michel Braudeau, Livres. "E.T.A. Hoffmann l'extraterrestre", EX, 12.8.1983, S.21.

Ralph de Gubernatis, "La danse de la mort de Kurt Jooss", NO, 26.11.1983, S.104-105 ; "Le document de la semaine: Günter Grass, Nicaragua ya!", NO, 1.1.1983, S.57; "Philosophie. Le combat serein avec Heidegger (Patrick Loriot, Martin Heidegger, L'Herne), NO, 23.12.1983, S.67.

Philippe Boyer, "Lettres allemandes: quand ils entendent le mot Kultur", NO, 17.6.1983, S.78; ders. "Conte expertise: si l'Allemagne nous était contée...", NO, 18.3.1983, S.90 91; Nita Rousseau, "Lettres allemandes: les larmes amères d'Irmgard Keu", NO, 6.5.1983, S.104. 
„Kind des Volkes“ (L'Express) suchen die Deutschen eine „Bestätigung für Pazifismus und Nationalismus" (Le Nouvel Observateur). Der nationalsozialistischen Vergangenheit werden jeweils neun Artikel gewidmet. Der fünfzigste Jahrestag von Hitlers Machtergreifung ist Titelgeschichte des Nouvel Observateur vom 28. Januar mit Analysen von Manès Sperber, Jean Daniel und Gérard Sandoz. ${ }^{47}$ Alfred Grosser schreibt über „Die Geschichte und das Geld ${ }^{48}$, Philippe Aubert berichtet über Klaus Gerstein, der nach Kriegsende über die Gaskammern informierte. ${ }^{49}$ In L'Express untersuchen Fred Kupfermann und Raymond Aron die Lage in Deutschland zu Beginn der dreißiger Jahre. ${ }^{50}$ Die Entdeckung des Chefs der Gestapo von Lyon Klaus Barbie wird ausführlich kommentiert: in den Titelgeschichten am 11. Februar und in weiteren Artikeln. ${ }^{51}$

Insgesamt ist das Deutschlandbild 1983 in Le Nouvel Observateur von einem gewissen Misstrauen geprägt. Es zeigt ein Land mit neutralistischen Tendenzen, welches wirtschaftliche Schwierigkeiten und soziale Spannungen bedrohen. In L'Express ist das Bild weniger düster. Die Journalisten teilen

46 Philippe Schneider, "Luther: la naissance du singulier", EX, 8.4.1983, S.24; Alain de Penaster, "Martin Luther: fils du peuple", EX, 2.12.1983, S.54 ; François Schlosser, "Luther mit uns", NO, 18.11.1983, S.47 ; Jean Delumeau, "Le chef d'œuvre de la semaine: Martin Luther von Cranach d $\mathrm{A}, N O, 26.11 .1983$, S.56.

Titelgeschichte: "Hitler pourquoi?", NO, 28.1.1983; Gérard Sandoz/ Jean Paul Enthoven, "Le jour où Adolf Hitler a gagné", Interview mit Manès Sperber, NO, 21.1.1983, S.90 ; Gérard Sandoz, "Nazisme: étaient-ils fous, ces Allemands?", ebd., S.36- 38; Jean Daniel, "Leur Allemagne", ebd., S.20.

Alfred Grosser, "L'Histoire et l'argent", EX, 29.4.1983, S.109. Philippe Aubert, "Allemagne: le SS de Dieu", L'Express, 18.3.1983, S.30. von Jean-Louis Missika und Dominique Wolton mit Raymond Aron, ebd., S.100-105.

Titelgeschichte "Barbie: le tribunal de l'histoire", L'Express, 11.2.1983; Raymond Aron, "Affaire Barbie: crimes contre l'humanité", ebd., S.70-72; Jacques Derogy, "La longue traque", ebd., S.73-76; Jérôme Dumoulin, "Elysée: opération Barbie", ebd., S.77-78 ; Sylviane Stein, "Ces nazis qu'on cherche encore", ebd., S.81-82; "Affaire Barbie: les masques de Vergès", 18.11.1983, S.118-120; Titelgeschichte "Klaus Barbie: la vérité quarante ans après", NO, 11.2.1983 mit Artikeln von Jacques Julliard, I. Allier, Elisabeth Schemla, Franz Olivier Giesbert, Georges Mamy, Ania Francos ; I. Allier, "Extradition: des juges français pour Klaus Barbie?", NO, 4.2.1983, S.33. 
zwar das Unbehagen hinsichtlich der Friedensbewegung, schätzen jedoch die wirtschaftliche Lage optimistischer ein.

1984

Im Jahr 1984 erscheinen weniger Artikel über Deutschland und die deutsch-französischen Beziehungen als im Vorjahr, zweiundneunzig im Vergleich $\mathrm{zu}$ hundertdreizehn, davon fünfundfünfzig in L'Express und siebenunddreißig in Le Nouvel Observateur. Beide Zeitungen veröffentlichen jedoch ausführliche Dossiers über das Nachbarland.

Das Dossier von Le Nouvel Observateur stellt im Februar die Frage „Faut-il avoir peur des Allemands?“ (Müssen wir vor den Deutschen Angst haben? ${ }^{52}$ ), verneint sie aber letztlich. Einer Meinungsumfrage von Le Nouvel Observateur, France-Inter und Sofres zufolge findet mehr als jeder zweite Franzose die Deutschen sympathisch, nur noch jeder zehnte hält sie für unsympathisch. Jacques Julliard schreibt, ,ein anderes Deutschland entsteht unter unseren Augen“, und meint, dass diese Veränderungen die Franzosen nicht beunruhigen sollten. Robert Scipion zufolge sind die alten Schimpfwörter zur Kennzeichnung der Deutschen vergessen. ${ }^{53}$ Die Artikel des Dossiers thematisieren die Angst vieler Deutscher vor einem Atomkrieg und die damit verbundene Suche nach einer Verantwortungsgemeinschaft mit dem zweiten deutschen Staat. Sie betonen auch den Wunsch nach einer neuen nationalen Identität, welche die „Wohlstandssymbole der sechziger Jahre“ durch ein „grünes Kulturideal“ ersetzt. ${ }^{54}$ Die Herausgeberin der Wochenzeitung Die Zeit, Marion Gräfin Dönhoff, versucht, die Ängste der

52 Dossier "Faut-il avoir peur des Allemands?", NO, 10.2.1984 : Jacques Julliard, "Faut-il avoir peur des Allemands?", ebd., S.40-43 ; Marie Muller, "Nous voulons gagner la paix", ebd., S.44-46 ; Gérard Sandoz, "Un pays soudain devenu vieux...", ebd., S.46 ; ders., "Toujours la plus forte armée d'Europe", ebd., S.47; ders., "La longue marche du S.P.D.", ebd., S.49-50; François Schlosser, "Plus fort que le mur", ebd., S.48; Rudolf von Thadden, "La faute à Luther", ebd., S.47 ; Robert Scipion, "Siegfried et la Limousine", ebd., S.50; François Schlosser/ Ruth Valentini, Interview mit Fritz Raddatz: "Le désespoir glacé de notre littérature", ebd., S.51 ; André Glucksmann, "A une amie allemande...", ebd., S.52; Marion Gräfin Dönhoff, "A un ami français...", ebd., S.53.

53 Robert Scipion, "Siegfried et la Limousine", NO, 10.2.1984, S.50. 
Deutschen verständlich zu machen, der Philosoph André Glucksmann erörtert die Angst der Franzosen vor deutschen Ängsten. Diese spiegelbildlich angelegten und in Briefform verfassten Artikel sind „An einen französischen Freund" und „An eine deutsche Freundin“ gerichtet. Im Nouvel Observateur führt zudem der Zeit-Redakteur Fritz Raddatz den Pessimismus der deutschen Gegenwartsliteratur darauf zurück, dass ein Dritter Weltkrieg im Bereich des Möglichen liegt und er Deutschland auslöschen würde.

Das Dossier von $L^{\prime}$ Express erscheint im Oktober unter dem Titel „Spécial Allemagne. Une nation dans ses Etats“ (Deutschland. Eine Nation in ihren Staaten) und kommentiert auf knapp hundert Seiten ebenfalls den Wandlungsprozess der Bundesrepublik. Es werden auch elf deutsche Persönlichkeiten vorgestellt, die alle, mit Ausnahme des Schriftstellers Heinrich Böll und des Verlegers Axel Springer, aus Politik und Wirtschaft kommen. Es handelt sich um Bundesaußenminister Hans-Dietrich Genscher, Sozialminister Norbert Blüm, den Ministerpräsidenten von BadenWürttemberg, Lothar Späth, den Ministerpräsidenten des Saarlandes Oskar Lafontaine, den Vorsitzenden des Deutschen Gewerkschaftsbundes Franz Steinkühler, den Industriellen Friedrich Karl Flick, Bundesbankpräsident Karl Otto Pöhl, den Präsidenten des Deutschen Industrie- und Handelstages Otto Wolff von Amerongen sowie den ehemaligen sozialdemokratischen Finanzminister Manfred Lahnstein. In Interviews kommen zu Wort: Edgar Reitz, der Autor des Films Heimat, Peter Stein, der Direktor der Berliner Schaubühne und Bundespräsident Richard von Weizsäcker. ${ }^{55}$

Der Sicherheitsexperte der SPD Egon Bahr betont die Notwendigkeit der Entspannung und wendet sich gegen eine Neutralisierung der Bundesrepublik, da sie das europäische Gleichgewicht stören würde. Der CSU-Vorsitzende und bayerische Ministerpräsident Franz Josef Strauß unterstreicht die Notwendigkeit einer „Politik der kleinen Schritte“ im Verhältnis zum Ostblock und zur Verbesserung der innerdeutschen

55 Dossier "Spécial Allemagne. Une nation dans ses Etats", EX, 19.10.1984; Guillaume Malaurie, Interview mit Edgar Reitz, "A la recherche de l'identité allemande", ebd., 1984, S.42-43; Mathieu Galey, Interview mit Peter Stein, "La névrose allemande", ebd., S.45; Interview mit Richard von Weizsäcker, "Le credo du président von Weizsäcker", ebd., S.21. 
Beziehungen. ${ }^{56}$ Den Schwerpunkt des Dossiers bilden Artikel zu Wirtschaft und sicherheitspolitischen Fragen sowie über den zweiten deutschen Staat. ${ }^{57}$ Patrick Bonazza stimmt einem hohen Beamten im Bonner Wirtschaftsministerium zu, demzufolge die Deutschen einen ,natürlichen Hang zum Pessimismus" haben. Denn die Lage sei keineswegs hoffnungslos, auch wenn die deutsche Industrie bei Spitzentechnologien etwas ins Hintertreffen geraten sei. Der Lebensstandard der Bundesdeutschen ist nach wie vor einer der höchsten der Welt, die Autoindustrie erfreut sich guter Gesundheit, die deutsche Wirtschaft beherrscht den europäischen Markt. Anne-Marie Le Gloannec informiert ausführlich über das Schwanken der SPD zwischen Technologie und Ökologie, Marktwirtschaft und staatlicher Wirtschaftsplanung, Treue zum Atlantischen Bündnis und Flirt mit dem Neutralismus. ${ }^{58}$ Olivier Wormser untersucht die innerdeutschen Beziehungen, Joseph Rovan die unterschiedlichen Interpretationen der deutschen Staatsangehörigkeit in Bonn und Ostberlin. Er gibt auch einen Überblick über die deutsche Geschichte von ihren Anfängen bis heute. ${ }^{59}$ Alfred Grosser teilt nicht die Sorge mancher Franzosen, die deutsch-französischen Beziehungen würden zugunsten der deutsch-deutschen Beziehungen vernachlässigt. ${ }^{60}$ Artikel über Theater, Fernsehen, Musik und bildende Kunst runden das Bild ab. Pierre Schneider informiert über die Maler König, Baselitz, Kiefer und Immendorff. ${ }^{61}$

56 Egon Bahr, "Priorité à la détente", ebd., S.25-27; "Franz-Josef Strauss: Une politique des petits pas", ebd., S.25-27.

57 Patrick Bonazza, "Economie: le syndrome du retard", EX, 19.10.1984, S.35-37; ders., "R.D.A.: les soviets plus l'électronique", ebd., S.37; Jérôme Dumoulin, "Allemagne de l'Ouest en première ligne", ebd., S.31-33; Michèle Georges, "Choses vues en R.D.A.", ebd., S.28-30; dies.,"Les cousins pauvres de l'Est", ebd., S.40; Michel Meyer, "Profession: marchand d'âmes" (Porträt des DDR-Anwalts Wolfgang Vogel), ebd., S.92-93. Anne-Marie Le Gloannec. "S.p.d.: le recentrage ambigu", EX, 19.10.1984, S.99-121.

Olivier Wormer, "Jusqu'où peut-on aller trop loin?", EX, 19.10.1984, S.34; Joseph Rovan, "Une ou deux citoyennetés?", EX, 19.10.1984, S.24-25; ders., "Empereurs, princes et chanceliers", ebd., S.22-23. Alfred Grosser, "Une nation dans ses Etats", EX, 19.10.1984, S.18-20. 
Zur Innenpolitik veröffentlicht L'Express elf Artikel, Le Nouvel Observateur acht. Das geringere Interesse an diesem Themenbereich als im Vorjahr, vor allem in Le Nouvel Observateur (acht Artikel im Vergleich zu sechsundzwanzig!), liegt in erster Linie an den reduzierten Aktivitäten der Friedensbewegung nach Ratifizierung des NATO-Doppelbeschlusses. Gleichwohl befürchten auch 1984 beide Zeitungen ein Abdriften der Bundesrepublik in die Neutralität und sie kritisieren ein zu starkes Interesse an Mitteleuropa. Nur L'Express behandelt die Affäre um einen der Homosexualiät bezichtigten Viersternegeneral ${ }^{62}$ und informiert über die Bundeswehr: Trotz fehlender Traditionen bildet sie die "Speerspitze der NATO“. ${ }^{63}$ Der „Ansturm“ der dreißigtausend „sehr lästigen“ Aus- und Übersiedler aus Osteuropa interessiert ebenso wie die finanziellen Leistungen der DDR für (ledige) Mütter, die eine hohe Geburtenrate zur Folge haben, trotz weitgehender Freigabe der Abtreibung. ${ }^{64}$ Das Streben des zweiten deutschen Staates nach mehr Unabhängigkeit von Moskau zugunsten der innerdeutschen Beziehungen wird thematisiert, desgleichen Maßnahmen zur Terrorismusbekämpfung und, wie bereits im Vorjahr, die Flick-Affäre (Parteispenden). Der deutsche Journalist Klaus-Peter Schmid zeichnet zudem das Porträt des neu gewählten Bundespräsidenten Richard von Weizsäcker. ${ }^{65}$

ebd, S.49-51; Michel Meyer, "Une télé de toutes les couleurs", ebd., S.41-43. M. Meyer erörtert die Funktionsweise des öffentlich-rechtlichen Rundfunks. Die regionale Verankerung der ARD (Arbeitsgemeinschaft der öffentlich-rechtlichen Rundfunkanstalten der Bundesrepublik Deutschland) entspreche einem Bedürfnis nach Heimat. Christian Hocke/ Anne Marie Fiel, "R.F.A.: l'honneur d'un général", EX, 20.1.1984, S.28.

Anne-Marie Le Gloannec/ Michel Meyer, "Armée R.F.A.: le refus de la tradition", EX, 16.3.1984, S.132-138; Michel Meyer, "Le fer de lance de l'Otan", ebd., S.138-142.

"Allemagnes: du bon usage des réfugiés", EX, 6.4.1984, S.32-33; Michèle Georges, "Allemagnes: la ruée vers l'Ouest", EX, 27.4.1984, S.46-47; "Allemagne: des réfugiés très encombrants", EX, 6.7.1984, S.21; Michèle Georges, "Allemagne de l'Est: chers bébés", EX, 3.8.1984, S.16.

Jérôme Dumoulin, "Allemagne: les humeurs de Moscou", EX, 10.8.1984, S.31; Michel Meyer, "R.F.A.: le séisme de l'affaire Flick", $E X, 6.10 .1984$, S.37; "R.F.A.: avec les commandos antiterroristes", EX, 9.11.1984, S.228-233; Klaus-Peter Schmid, "Portrait: Richard von Weizsäcker au-dessus de la mêlée", EX, 9.3.1984, S.114-115. 
In Le Nouvel Observateur gibt Gérard Sandoz einem jungen ostdeutschen Schriftsteller, der anonym bleiben möchte, die Möglichkeit, den Militarismus der DDR zu denunzieren und die Bedeutung der Kirchen in der Friedensbewegung hervorzuheben. Der Journalist betont auch den Wunsch Ost-Berlins nach engerer Zusammenarbeit mit Westeuropa und meint, die innerdeutschen Beziehungen waren noch nie so gut wie in diesem Jahr, in dem beide deutsche Staaten ihre Verantwortungsgemeinschaft für den Frieden erkennen. ${ }^{66}$ Weitere Themen sind die Schiedsrichterrolle der Grünen bei der Europawahl und das Tief des Bundeskanzlers: Ein Skandal pro Monat unterhöhlt seine Autorität, um so mehr, als er eine moralische Wende versprochen hatte. ${ }^{67}$

In Bezug auf die deutsch-französischen Beziehungen erklärt Jacques Julliard in Le Nouvel Observateur, die ursprünglich „sehr zerbrechliche Vernunftehe" halte inzwischen erstaunlich gut. Einige Zeilen weiter vergleicht er aber beide Länder im Strategiebereich mit Zügen, die in unterschiedliche Richtungen fahren. ${ }^{68}$ Während Frankreich endlich die Notwendigkeit einer europäischen Verteidigung einsieht, strebt Deutschland nach mehr Unabhängigkeit vom Atlantischen Bündnis zwecks besserer Beziehungen zum Ostblock. ${ }^{69}$ Der Historiker Rudolph von Thadden erörtert das komplexe Verhältnis der deutschen Intellektuellen zu Europa. ${ }^{70} L^{\prime}$ Express setzt teilweise andere Schwerpunkte. Kommentare zu Gunsten einer europäischen Verteidigung werden ergänzt durch Informationen über die Abschaffung der

66 Gérard Sandoz, "Allemagnes: à l'Est, du nouveau", NO, 20.1.1984, S.34-35; ders., "BerlinEst: le grand déménagement", $N O$, 20.4.1984, S.39; ders., "Allemagne: par-dessus le mur", NO, 27.7.1984, S.34; ders., "Allemagnes: Berlin-Est fronde, Moscou gronde", NO, 10.8.1984; ders., "Cette Europe qui nous manque. R.D.A.: des coups sur le mur", NO, 17.8.1984, S.28. ; ders., "Allemagne: Honecker en retenu", NO, 7.9.1984, S.42. chancelier à marée basse", $N O, 6.7 .1984$, S.34. Jacques Julliard, "Faut-il avoir peur des Allemands?", NO, 10.2.1984, S.31. vieux continent", $N O, 16.3 .1984$, S.38-39. 
Grenzkontrollen zwischen Deutschland und Frankreich im Rahmen der Schengener Abkommen. ${ }^{71}$

Deutsche Außenpolitik, soweit sie den europäischen Rahmen und die Ost-West-Beziehungen überschreitet, interessiert 1984 nicht. Zum Thema Wirtschaft finden wir in L'Express zwei Artikel, drei in Le Nouvel Observateur. Beide kommentieren die Forderung des Deutschen Gewerkschaftsbundes nach einer 35-Stunden-Woche sowie geplante Streikmaßnahmen. L'Express erörtert zudem den Strukturwandel der deutschen Industrie und unterstreicht, dass Frankreich ähnliche Probleme hat. Wie kann die Wirtschaft modernisiert werden, ohne dass die Arbeitslosigkeit steigt? ${ }^{72}$ Was Entwicklungen der Gesellschaft betrifft, so untersucht Le Nouvel Observateur die Begeisterung der Deutschen für Straßenfeste. ${ }^{73}$ L'Express analysiert die Identitätskrise der ,immer noch geteilten Nation“, die Rolle der Türken in der Bundesrepublik sowie Entwicklungen in der DDR. ${ }^{74} \mathrm{Im}$ Kulturbereich berichten beide Zeitungen über Pina Bausch sowie den Direktor der Berliner Schaubühne Peter Stein. ${ }^{75}$ Sie würdigen auch die Filmkunst von Fritz Lang. Le Nouvel Observateur verfolgt darüber hinaus

71 Jérôme Dumoulin, "Défense européenne: Paris ranime la flamme", EX, 24.2.1984, S.41; Nicole Gnesotto, "Défense européenne: histoire d'une première française", $E X, 13.7 .1984$, S.44 ; "France-Allemagne: jeux sans frontières", EX, 13.7.1984, S.23.

72 Gérard Sandoz, "Allemagne: I.G.Metall, le défi des trente-cinq heures", NO, 2.3.1984, S.31; ders., "R.F.A.: une grève explosive", NO, 25.5.1984, S.25 ; François Schlosser, "Allemagne: la croisade des trente-cinq heures", $N O, 11.5 .1984$, S.51;

Michel Meyer, "Allemagne: la croissance quand-même", EX, 10.2.1984, S.19; Michel Jacques, "Les mutations, version allemande", EX, 13.4.1984, S.32-33 ; ders., "R.F.A.: le Kriegsspiel des métallos", EX, 25.5.1984, S.26-27.

Alain Schifres, "R.F.A.: Schwarzenbeck, le dos au mur", NO, 8.6.1984, S.52.

"Document. R.F.A.: Crise d'identité dans une nation toujours divisée"; Anne-Marie Le Gloannec, "Le mal allemand", EX, 27.1.1984, S.94-100; Michèle Georges, "Allemagne: le pouvoir turc", EX, 18.5.1984, S.32-33; Joseph Rovan, "L'Allemagne selon Marx", EX, 28.12.1984, S.44-45. "Théâtre: Berlin, allez les Français!", EX, 4.5.1984; "Théâtre: Peter Stein, Saint-Just saisi par les doutes", EX, 12.10.1984, S.145; Guy Dumur, "Scène : les enfants de Pina Bausch et de Shakespeare", NO, 13.1.1984, S.61; Ruth Valentini, "Danse: la fée de Wuppertal", NO, 15.6.1984, S. 86-88; Guy Dumur, "Théâtre: les Allemands entre chien et loup" (Syberberg, Stein, Grüber, Heiner Müller), NO, 12.10.1984, S.114-118. 
die Diskussion um die Frankfurter Aufführung des Theaterstückes von Rainer Maria Fassbinder Der Müll, die Stadt und der Tod. Karl Poppers Analyse der Freiheit des Menschen wird besprochen, ${ }^{76}$ die Lessing- und KleistInszenierungen von Giorgio Strehler am Pariser Théâtre de l'Europe werden kommentiert, desgleichen Ausstellungen von Adolf Menzel und Paul Klee sowie Publikationen über Franz Kafka und Ernst Jünger. ${ }^{77}$ L'Express informiert ebenfalls über deutsche Kultur in der französischen Hauptstadt: Lessing im Théâtre de 1'Europe, Wagner im Palais Garnier, Kafka im Centre Pompidou, Altdorfer im Centre Culturel du Marais. Artikel über Arnold Böcklin und Johann Sebastian Bach ergänzen das Bild. ${ }^{78}$

Beide Pressorgane problematisieren die nationalsozialistische Geschichte. Neben ausführlichen Kommentaren zum vierzigsten Jahrestag der Befreiung Frankreichs im Juni 1944 erscheinen Buchbesprechungen, darunter die französische Übersetzung von Schindler Liste. ${ }^{79}$

\section{5}

76 Gérard Sandoz, "Allemagne: l'affaire Fassbinder", NO, 3.8.1984, S.27 ; Didier Eribon, "Karl Popper: 'oui, nous sommes libres', $N O, 14.9 .1984$, S.95.

77 Paul Thevenon, "Fritz Lang: la jouvence du Dr. Moroder", EX, 10.8.1984, S.38; Gérard Sandoz, "Gustav Fröhlich: je suis enchanté..."/ "Un rocker nommé Fritz Lang", NO, 27.7.1984; M. Mardore, "Rétrospective: l'ami Fritz", NO, 16.11.1984, S.105; Guy Dumur. "Théâtre: la bonne âme de Saxe", NO, 6.1.1984; ders., "Théâtre: les sables noires du Brandebourg", ebd., 1.7.1984, S.78 ; ders., "Hommage: faut-il adorer Kafka?", NO., 22.6.1984, S. 75 ; "Exclusif: une lettre inédite de Franz Kafka", ebd., S. 76 ; Werner Hofmann, "Mur d'atelier d'Adolf Menzel", NO, 16.11.1984, S.102 ; France Huser, "Klee, ombre et silence" (Ausstellung im Museum der Kunstakademie von Nîmes), NO, 24.8.1984, S.81 ; Jean-Paul Enthoven, "Dans l'œil de Jünger", ebd., 17.8.1984, S.48-49.

Mathieu Galey, "Théâtre: connaissez-vous Lessing?", EX, 13.1.1984, S.68; "Opéra : Tannhäuser repêché", EX, 20.7.1984, S.66; Pierre Schneider, "Altdorfer: le crépuscule de Dieu", EX, 20.4.1984, S.112; Paul Thevenon, "Kafka multiplié par trois", EX, 5.10.1984, S.134; Pierre Schneider, "Quand les peintres allemands peignaient français", $E X, 2.11 .1984$, S.119; "Bach sans perruque", EX, 28.12.1984, S.66-67.

Michèle Georges, "Hitler, le faussaire et les pigeons", EX, 14.9.1984, 55-56; Fred Kupfermann, "Portrait de Hitler en dynamo", ebd., 23.11.1984, S.179; Pierre Vidal-Naquet, "Nazisme: le secret partagé", ebd., 80-81; Eugen Kogon, Les chambres à gaz, secret d'Etat, Les Editions de Minuit, NO, 21.9.1984; F.Ciavigliolo, "Bienfaisance: le nazi de Jérusalem" (Thomas Keneally, La liste de Schindler, Robert Laffont), ebd., 2.3.1984, S.88; Didier Eribon, "Rencontres: Albert Hirschman de Berlin à Princeton", NO, 8.6.1984, S.85. 
Das Jahr 1985 ist geprägt von deutsch-französischen Gemeinschaftsprojekten im Technologie- und Verteidigungsbereich. Am 17. April 1985 übermittelt Außenminister Roland Dumas den EG-Partnern den Vorschlag des französischen Präsidenten François Mitterrand, ein „Europa der Technologie“ zu schaffen, um ,unserem Kontinent die Beherrschung aller Spitzentechnologien $\mathrm{zu}$ ermöglichen und ihn zum Kontinent des einundzwanzigsten Jahrhunderts werden zu lassen".80 Die deutschen Reaktionen auf diesen Vorschlag werden ausführlich kommentiert. Ein weiteres wichtiges Thema ist der Weltwirtschaftsgipfel von Bitburg (2.-4. Mai 1985) mit dem daran anschließenden Besuch von Bundeskanzler Helmut Kohl und US-Präsident Ronald Reagan auf dem dortigen Soldatenfriedhof, auf dem auch Angehörige der Waffen-SS begraben sind. Helmut Kohl wollte damit anlässlich des vierzigsten Jahrestags der deutschen Kapitulation die Aussöhnung zwischen Deutschland und den Vereinigten Staaten medienwirksam demonstrieren, löste dabei aber starke Kritik aus. Bernard Ullmann und Michel Meyer werfen dem Kanzler vor, die Geister der Vergangenheit $\mathrm{zu}$ wecken und unterstreichen die „Unmöglichkeit des Vergessens". ${ }^{81}$ Insgesamt ist 1985 das Interesse an Deutschland in beiden Zeitungen deutlich geringer als 1984: fünfzig Artikel im Vergleich zu zweiundneunzig, einunddreißig in L'Express, neunzehn in Le Nouvel Observateur.

Die deutsche Innenpolitik erhält jeweils vier Artikel. L'Express behandelt Martin Bangemann als Nachfolger von Hans-Dietrich Genscher im FDP-Parteivorsitz, Oskar Lafontaines Wahlkampf im Saarland, Johannes Raus Wahlsieg in Nordrhein-Westfalen sowie Helmut Kohls Fähigkeit, Probleme auszusitzen..$^{82}$ Le Nouvel Observateur bevorzugt andere Themen: Forderungen der Vertriebenenverbände, Methoden des Bundeskriminalamtes zur

\footnotetext{
$80 \quad$ zitiert nach Dokumente 6/1987, S.529.

81 Bernard Ullmann/ Michel Meyer, "Allemagne: l'impossible oubli"/ "En ce temps là", $E X$, 26.4.1985, S.37; siehe auch das Interview mit Claude Lanzmann "Shoah: la mémoire infinie", EX, 10.5.1985, S.40.

82 Michel Meyer, "R.F.A. : nouveau patron pour parti usé", EX, 1.3.1985; ders., "Lafontaine nous voilà", $E X, 15.3 .1985$, S.42; ders., "R.F.A: la percée de Johannes Rau", EX, 17.5.1985, S.30-31; Elie Marcuse, "Un chancelier très coriace", EX,18.10.1985, S.45-47.
} 
Terrorismusbekämpfung, Spionage. ${ }^{83}$ Er verhält sich aber, wie L'Express, Oskar Lafontaine gegenüber reserviert, noch bevor dieser 1986 in die Debatte um das nahe der deutschen Grenze gelegene französische Kernkraftwerk Cattenom eingreift. Gérard Sandoz wirft ihm vor, seine Meinung nicht deutlich genug zu sagen. ${ }^{84}$

Der Gipfel von Bitburg zeigt Spannungen in den deutschfranzösischen Beziehungen. Der Bundeskanzler unterstützt nicht die Forderung des französischen Präsidenten, die von den USA geforderte neue GATT-Runde an eine Reform des internationalen Währungssystems zu koppeln. Zu weiteren Meinungsverschiedenheiten führt das amerikanische SDI-Projekt (Strategic Defense Initiative), das Frankreich, anders als die Bundesrepublik nicht befürwortet. In L'Express bezeichnet deshalb JeanClaude Casanova den Gipfel als ,überflüssig“ und spricht von einer „Abkühlung“ zwischen Paris und Bonn. ${ }^{85}$ Jérôme Dumoulin unterstreicht die von Paris unterschätzte Manövrierfähigkeit Helmut Kohls, mit der er die amerikanische und die europäische Karte gleichzeitig ausspielt. ${ }^{86}$ Le Nouvel Observateur sieht Bonn und die anderen Europäer stärker als L'Express in der Rolle von „Verrätern“. François Schlosser bedauert, dass die Bundesrepublik nicht bereit sei, eigene Interessen zugunsten Europas zurück zu stellen. ${ }^{87}$ Auch in den folgenden Monaten bestimmen Sicherheitsfragen und die Suche nach einer europäischen Antwort (,Eurêka“) auf die amerikanischen Strategiepläne SDI die L'Express-Berichterstattung. Elie Marcuse zufolge zeigte der Gipfel von Bitburg, dass das deutsch-französische Tandem ,platt“ sei. Man müsse von einem „deutsch-französischen Unbehagen“ sprechen, obwohl Helmut Kohl und François Mitterrand vereinbarten, bis zum EG-Gipfel in Mailand

83 Gérard Sandoz, "Allemagne: à nous, la Silésie!", NO, 18.1.1985, S.34; ders., "Allemagne: les armes du B.K.A". Interview mit Heinrich Boge (Leiter des Bundeskriminalamtes), NO, 8.2.1985, S.30; Claude Raffin, "Allemagne: le bal des espions", NO, 30.8.1985, S.32-34.

Gérard Sandoz, "Les caprioles d’Oscar Lafontaine", NO, 15.3.1985, S.51.

Jérôme Dumoulin, "Kohl: le prix des étoiles", EX, 24.5.1985, S.35-36 ; ders., "Bonn-Paris: le coup de froid", EX,10.5.1985, S.33-36; Jean-Claude Casanova, "Un sommet inutile et incertain", ebd., S.37.

86 Jérôme Dumoulin, "Kohl: le prix des étoiles", EX, 24.5.1985, S.35.

François Schlosser, "Europe. Bonjour les traîtres", NO, 10.5.1985, S.36. 
mehr Gemeinsamkeiten zu erarbeiten. ${ }^{88}$ Altbundeskanzler Willy Brandt nimmt Ende Juni Stellung zu Europas Zukunft. Gemeinsame Technologieprojekte reichen nicht aus, es gilt, ein überzeugendes europäisches Sozialprogramm zu entwickeln. ${ }^{89}$ Patrick Bonazza, José Alain Fralon und Elie Marcuse äußern im weiteren Verlauf des Jahres erneut die Sorge, Europas Zukunft werde verspielt, falls die Bundesrepublik sich nicht stärker an gemeinsamen Technologie- und Verteidigungsprojekten beteilige. Paris könne sich zu wenig auf Bonn verlassen, nicht einmal beim geplanten deutsch-französischen Kampfhubschrauber. ${ }^{90}$ Anfang Dezember wird konstatiert: „Paris sieht jeden Tag die Liste der Misserfolge bei den Gemeinschaftsprojekten länger werden: nach dem Panzer das Kampfflugzeug, der Beobachtungssatellit und vielleicht der Panzerabwehrhubschrauber". ${ }^{91}$ In Le Nouvel Observateur kritisiert Gérard Sandoz unter der Überschrift „Essigmond zwischen Bonn und Paris“ das geringe Interesse Bonns am Eurêka-Projekt: „Das alte Gründungspaar der europäischen Einheit umarmt sich zwar noch“, doch „das Herz ist nicht mehr dabei“. Die Beziehungen hätten sich seit 1983 verschlechtert, seit der „gemeinsamen Schlacht“ um die Stationierung der amerikanischen Mittelstreckenraketen, in welcher Mitterrands Rede vor dem Bundestag eine „schöne Demonstration deutsch-französischer Einstimmigkeit“ darstellte. ${ }^{92}$

Deutsche Außenpolitik thematisiert Le Nouvel Observateur nur im Rahmen des Gipfels von Bitburg, L'Express erörtert zudem Willy Brandts Initiativen zur „Verbesserung der deutsch-polnischen Beziehungen“.93 Nur

88 Michel Meyer, "France-R.F.A.: les divergences de Constance", EX, 31.5.1985, S.46-47; Elie Marcuse, "Le tandem franco-allemand à l'épreuve", EX, 28.06.1985, S.34-35.

"L'avenir de l'Europe vu par Helmut Kohl et Willy Brandt", EX, 28.6.1985.

Elie Marcuse, "Un bouclier européen?", EX, 12.7.1985, S.15 ; ders., "Eurêka: le deuxième pas qui coûte", $E X, 26.7 .1985$, S.24 ; ders., "Avion de combat: la France en solo", EX, 16.8.1985, S.36-37; "Europe: Airbus. Le forcing allemand", EX, 8.2.1985, S.38 ; José Alain Fralon, "Europe: la relance gâchée", EX, 5.7.1985.

Elie Marcuse/ Patrick Bonazza, Y.C., "Relance européenne", EX, 6.12.1985, S.37.

Gérard Sandoz, "Paris-Bonn: lune de vinaigre", NO, 1.3.1985, S.40. Siehe auch: François Schlosser, "Europe: le consensus secret d'Helsinki", NO, 29.3.1985, S.40 ; Josette Alia, "Stratégie: l'Europe du ciel", NO, 26.4.1985.

Elie Marcuse, "R.F.A.-Pologne: le jeu bizarre de Willy Brandt", EX, 13.12.1985, S.46. 
L'Express informiert über den neuen Wirtschaftsaufschwung. ${ }^{94}$ Zum Thema Gesellschaft schreibt Michel Meyer, dass die Bundesdeutschen nach Jahren der Angst eine positive, sehr materialistische Lebenseinstellung entwickelt haben. ${ }^{95}$ Fünf Artikel in L'Express beschäftigen sich mit Kultur. Sie kommentieren den Tod des Verlegers Axel Springer, die Erfolge des Filmemachers Wim Wenders in Japan, die französische Übersetzung der Nietzsche-Biografie von Curt Paul Janz sowie die Klee-Ausstellung im Pariser Centre Pompidou. ${ }^{96}$ Simone Dupuis informiert über das Münchner Gastspiel des Balletts von Maguy Marin. ${ }^{97}$ Le Nouvel Observateur interessiert sich 1985 wenig für deutsche Kultur, aber Wim Wenders und die Pariser KleeAusstellung werden erwähnt. Zudem erscheint ein Artikel über Kant.98 L'Express thematisiert Geschichte anlässlich der Entdeckung des Grabes des berüchtigten Arztes von Auschwitz, Josef Mengele, in der Buchbesprechung „Hitlers amerikanischer Freund“ und einem Artikel über „Nazikitsch“"99. In Le Nouvel Observateur bespricht der Philosoph Alain Finkielkraut den Tagungsband des von Raymond Aron und François Furet geleiteten Kolloquiums an der Sorbonne zum Thema „Nazideutschland und der Völkermord an den Juden“. ${ }^{100}$ Nina Sutton und Gérard Sandoz gehen in einem Interview mit Josef Peter Stern von der Universität London der Frage nach: „Wer hat Schuld an Hitler?“ Der geplante Prozess gegen den Chef der Gestapo

94 Michel Jacques, "Economie. R.F.A.: la montée en puissance", EX, 8.3.1985, S.39.

95 Michel Meyer, "Le tiroir-caisse anti-déprime", EX, 25.1.1985, S.36.

96 Elie Marcuse, "Un empereur s'en va", EX, 27.9.1985, S.49; "Wim Wenders: le Japon évanoui", $E X, 22.11 .1985$, S.142; J.Bollon, "L'odyssée du moi", EX, 21.6.1985, S.118; P. Schneider über die Klee-Ausstellung im Centre Pompidou, EX, 25.10.1985, S.125. Simone Dupuis, "Danse: la France envahit l'Allemagne", EX, 15.11.1985, S.134. M.Mardore, "Culte: Wenders célèbre le rite Ozu", NO, 15.11.1985, S.96 ; Denis Roche, "Paul Klee : 'Harmonisierter Kampf'”, NO, 13.9.1985, S.76-77. Jean-Paul Enthoven, "Un Kant à soi. Kant entre la loi morale et l'embarras gastrique", $N O$, 12.7.1985, S.69. américain de Hitler", EX, 7.6.1985, S.156. "Nazisme: le kitsch au pouvoir", EX, 28.6.85, S.109.

100 Livres: Alain Finkielkraut, "Génocide: le bureaucratique et le fanatique", NO, 13.12.1985, S.72-73. 
von Lyon Klaus Barbie wird ausführlich kommentiert, ${ }^{101}$ desgleichen Claude Lanzmanns Film Shoah. Gérard Sandoz kritisiert zudem, dass nach Südamerika geflohene Nazis „ihrem Richter entkommen konnten“. ${ }^{102}$ Anders als L'Express erörtert Le Nouvel Observateur 1985 auch den dreihundertsten Jahrestag der Aufhebung des Edikts von Nantes und die damit verbundene Emigration französischer Hugenotten nach Preußen. ${ }^{103}$ Das große Interesse beider Zeitungen an der nationalsozialistischen Vergangenheit -ausgelöst durch den Besuch von Helmut Kohl und Ronald Reagan auf dem Soldatenfriedhof von Bitburg, die Entdeckung des Grabes von Mengele und den Film „Shoah“- bedeutet gleichwohl keine Fixierung auf dieses Thema.

1986

Anlässlich des „Frankfurter Kulturgipfels“ veröffentlichen Anfang November der deutsche und der französische Außenminister einen gemeinsamen Artikel in der Presse beider Länder. Hans-Dietrich Genscher und Jean-Bernard Raimond erklären in der Süddeutschen Zeitung (4.11.1986) und in Le Monde (5.11.1986), das Verhältnis zwischen Frankreich und Deutschland sei ,heute so beispielhaft, dass sich niemand mehr wundere, wenn ihre Außenminister gemeinsam zur Feder greifen" ${ }^{104}$ Gleichwohl ist 1986 das Jahr, in dem sowohl L'Express als auch Le Nouvel Observateur die wenigsten Artikel über Deutschland publizieren: siebenundvierzig, davon siebenundzwanzig in L'Express und zwanzig in Le Nouvel Observateur. Dies bestätigt, dass Presseorgane häufig an Konflikten und Spannungen interessiert

\footnotetext{
101 Nicole Sutton/ Gérard Sandoz, Interview mit Josef Peter Stern, "Hitler, la faute à qui?", NO, 12.4.1985, S.88 ; "Procès Barbie: Vergès parle", NO, 20.9.1985, S.72.

102 Claude David, "Nazisme: 'Shoah', la mémoire longue”, NO, 26.4.1985, S.74; Claude Lanzmann/Abraham Bomba, "Témoignage : 'Coiffeur' à Treblinka”, ebd., S.76; Claude Roy, "Le tribunal de la mémoire", ebd., S.77; Gérard Sandoz, "Crime de guerre: des nazis trop tranquilles", ebd., S.50.

103 "La Diaspora des papaillots", NO, 5.4.1985; Didier Eribon, Interview mit Rudolph von Thadden, "Pour le roi de Prusse", ebd., S.82. 
sind und positive Entwicklungen ihren Lesern weniger vermitteln - wie Alfred Grosser konstatierte. ${ }^{105}$

Innenpolitische Ereignisse und Entwicklungen interessieren 1986 wie bereits im Vorjahr wenig: drei Artikel in L'Express, zwei in Le Nouvel Observateur. L'Express kommentiert den Sieg der Konservativen bei den Wahlen in Niedersachsen, die Wahlniederlage der SPD in Hamburg ${ }^{106}$ und spricht wegen des Skandals um die gewerkschaftseigene Immobiliengesellschaft „Neue Heimat“ von der Notwendigkeit eines „Großreinemachens“ im Deutschen Gewerkschaftsbund. ${ }^{107}$ Le Nouvel Observateur berichtet ebenfalls über die „Neue Heimat“ sowie Vorschläge der Sozialdemokraten zum Thema Zweidrittelgesellschaft: Wie können Menschen vom Wohlstand profitieren, die vom Arbeitsprozess ausgeschlossen sind? ${ }^{108}$

In L'Express fordert der ehemalige französische Botschafter in Bonn Henri Froment - Meurice Frankreich auf, sich eindeutig zugunsten einer Verteidigung der BRD festzulegen und diese nicht länger als Puffer zu betrachten. ${ }^{109}$ Zwei Monate später konstatiert Elie Marcuse Schwierigkeiten in den deutsch-französischen Beziehungen und spricht von einem „misslungenen Dialog“ zwischen Bonn und Paris. Beide Länder redeten noch nie so viel über gemeinsame Sicherheit, doch die Prioritäten sind verschieden. ${ }^{110}$ Die Bereitschaft der Bundesregierung dreißig Prozent der Kosten des europäischen Raumgleiters Hermes zu tragen, löst Misstrauen aus: Françoise Harrois-Monin befürchtet, die Deutschen würden sich die

\footnotetext{
$105 \quad$ Siehe Anmerkung 1.

106 Michel Meyer, "Kohl sauvé par les Saxons", EX, 20.6.1986, S.36 ; Paul-Jean Franceschini, "R.F.A.: le SPD en cale sèche", EX, 14.11.1986, S.34.

107 "R.F.A.: la grande lessive syndicale", EX, 31.10.1986, S.49.

$108 \quad$ K.S. Karol, "Reportage. SPD: les aventuriers du tiers perdu", NO, 5.9.1986, S.60-62; Claude F.Jullien, "R.F.A.: les syndicats bradent", NO, 14.11.1986, S.54-55.

109 Henri Froment-Meurice, "France-R.F.A.: défense, sortons de l'ambiguïté", EX, 21.3.1986, S.53.

110 Elie Marcuse, "France-Allemagne: les ratés du dialogue", EX, 30.5.1986, S.28-29; ders., "Stratégie. L'Europe sur la défensive", EX, 5.12.1986, S.28.
} 
besonders lukrativen Verträge reservieren. ${ }^{111}$ In Le Nouvel Observateur kritisiert Alain Minc das „Finnlandsyndrom“, also vermeintlich neutralistische Tendenzen der Bundesrepublik. ${ }^{112}$ Im Rahmen der „Deutsch-französischen Begegnungen“ interviewt Jacques Leenhardt den Dramaturgen Heiner Müller. ${ }^{113}$

Nur L'Express kommentiert 1986 die deutsche Außenpolitik, und dies recht kritisch. Erneut stellt Michel Meyer die Frage nach der Verlässlichkeit Bonns als Bündnispartner und bemängelt, dass die Bundesregierung mit Rücksicht auf deutsche Exportinteressen in der arabischen Welt den amerikanischen Luftangriff auf Libyen nur halbherzig unterstützte. Die deutsche Diplomatie zeige „ewige Zweideutigkeiten“, die ihrerseits Folge der Versuchung seien, eine wirtschaftliche Supermacht darzustellen. ${ }^{1{ }^{14}}$ Der zweite Kommentar zur Außenpolitik behandelt die Währungspolitik und bedauert die unnachgiebige Haltung Bonns gegenüber Washington in der Zinsfrage, ${ }^{115}$ also die Weigerung, die deutschen Leitzinsen zugunsten einer Ankurbelung der amerikanischen Wirtschaft zu senken. Auch andere Aspekte der Wirtschaft finden nur in L'Express Beachtung. Elie Marcuse spricht von einem neuen Wirtschaftswunder und Michel Jacques fordert, die deutsche „Lokomotive“ nicht zu überfordern, da sie die Ökonomie Europas und die Weltwirtschaft in Schwung bringen soll. Zwar sind deutsche Unternehmen leistungsfähiger denn je, doch Bonn fürchtet einen Rückschritt. In ihrem Bericht über die deutsche Bekleidungsindustrie, die Frankreich überrundet und weltweit Platz zwei eingenommen hat, appelliert Marie-Pierre Gröndahl an die Franzosen mit verstärkter Kreativität auf diese Herausforderung zu reagieren. ${ }^{116}$ Auch Michel

\footnotetext{
111 Françoise Harrois-Monin, "L'Europe prend le taxi", EX, 31.10.1986, S.67-68.

112 "Europe: Pour en finir avec le syndrome finlandais", NO, 3.1.1986, S.40-41. Alain Minc ist Autor des Buches Le Syndrome finlandais, Seuil, 1986.

113 Jacques Leenhardt, Interview mit Heiner Müller: "Quels cousins germains?", NO, 14.2.1986, S.78-80.

114 Michel Meyer, "Bonn: oui, mais...", EX, 25.4.1986, S.34.

115 Jacques de Vernisy, "Dollar-Mark: l'heure des comptes", EX, 26.9.1986, S.29-30.

116 Elie Marcuse, "Bonn: le nouveau miracle", EX, 7.3.1986, S.45-46; Michel Jacques, "R.F.A.: ne poussez pas la locomotive", EX, 9.5.1986, S.23; Marie-Pierre Gröndahl, "Mode: la surprise allemande", $E X, 3.10 .1986$, S.49.
} 
Jacques empfiehlt deutsche Unternehmer Frankreich als Vorbild, denn sie geben dem Export die Priorität und halten Lieferfristen ein. ${ }^{1{ }^{17}}$ Insgesamt wird die Stärke der deutschen Wirtschaft unterstrichen und dabei entsteht der Eindruck, Frankreich gerate unter Druck. Entwicklungen der Gesellschaft beschreibt Elie Marcuse in seiner Analyse des „neue Wirtschaftswunders“: Deutschland möchte kein Land der Angst mehr sein, sondern wie der Tennisspieler Boris Becker als „Sieger in der internationalen Arena“ gelten. Sowohl zu Kultur als zur Rubrik Gesellschaft gehört ein Artikel von Le Nouvel Observateur, der München vorstellt: diese zugleich barocke und futuristische Stadt sucht ihren Weg zwischen „Nostalgie, Fußball und Kino“. ${ }^{118}$ Reaktionen auf Günter Wallraffs Buch Ganz unten (Tête de turc), das die Arbeits- und Lebensbedingungen türkischer Gastarbeiter in der Bundesrepublik aufdeckt, werden thematisiert. ${ }^{119}$ Mit neun Artikeln hat Kultur in L'Express die höchste Frequenz des Jahres 1986. Wir finden mehrere Buchbesprechungen: Günter Grass, Patrick Süskind sowie Alexandre Vialattes Erfahrungen in Deutschland zwischen 1922 und 1949. ${ }^{120}$ Die Berliner Filmfestspiele interessieren, desgleichen Ballet. Ein Artikel beschäftigt sich ausschließlich mit Pina Bausch, ein weiterer erwähnt sie in Zusammenhang mit der Tanzbiennale in Lyon. ${ }^{121}$ Die Leser werden informiert über den Berliner Maler Georges Grosz, Carl Maria von Webers Oper Oberon und

\footnotetext{
117 Michel Jacques, "Economie. La France vue d'Allemagne", EX, 27.6.1986, S.19-20.

118 Hervé Algalarrondo, "Munich baroque et futuriste, nostalgie, football et cinéma", $N O$, 26.12.1986, S.69.

119 Gilles Perrault, "Zoro est allemand", NO, 28.3.1986, S. 92 ; Interview mit Klaus Schuffels, "Allemagne. L'effet Wallraff", NO, 31.10.1986, S.84-85.

120 "Livres. Qoui de neuf? L'Allemagne", Pascal Guignard bespricht Patrick Süskind, Nicole Casanova Günter Grass, EX, 11.4.1986 ; dies., "Livres. Allemagne: l'harmonie perdue", EX, 21.2.1986, S.136-137; Littérature: A.R., Vialatte, "Choses vues en Allemagne", EX 10.1.1986, S.98.

121 François Forestier, "Cinéma: Berlin sera toujours Berlin", EX, 28.2.1986, S.98; Simone Dupuis, "Heureux qui voit Pina Bausch", EX, 23.5.1986, S. 42; dies., "Danse: tableau d'une exposition allemande", $E X, 12.9 .1986$, S.129-130.
} 
Strehlers Inszenierung der Dreigroschenoper von Brecht. ${ }^{122}$ Auch Le Nouvel Observateur würdigt Pina Bausch. Daneben erscheinen Artikel über Wilhelm Furtwängler, das Festival des Frauenfilms in Hamburg sowie den Bertelsmannkonzern, der doppelt so viel Umsatz erwirtschaftet wie der französische Medienriese Hachette. ${ }^{123}$ Berliner Kunstausstellungen werden kommentiert, Oswald Spenglers Preußentum und Sozialismus interessiert ebenso wie Gerhard Kierschs Untersuchung über die deutsche Jugend mit dem Titel Les héritiers de Goethe et d'Auschwitz (Die jungen Deutschen. Erben von Goethe und Auschwitz). ${ }^{124}$

Die nationalsozialistische Geschichte erörtern beide Wochenzeitungen vor allem mit Blick auf Österreich, anlässlich der Kandidatur von Kurt Waldheim für das Präsidentenamt. L'Express berichtet zudem über den HitlerStalin-Pakt von 1939 und die Kritik an der Errichtung eines Karmelitinnenklosters in Auschwitz. ${ }^{125}$ Die Besprechung von Elly Welts Josef sauvé par ses frères ${ }^{126}$ sowie ein Dossier über die französische Kollaboration ${ }^{127}$ ergänzen das Bild. Le Nouvel Observateur beschäftigt sich ebenfalls mit dem Hitler-Stalin-Pakt, ${ }^{128}$ dokumentiert Photographen in den

122 F. Erikson, "Musique: les richesses cachées de Carl Maria von Weber", EX, 30.5.1986, S.123; "Berlin au noir. Le peintre Georges Grosz (1893-1959)", EX, 5.8.1986; "L'événement Strehler", EX, 24.10.1986.

123 Henri-Louis de La Grange, "Furtwängler, le rescapé des pirates", NO, 24.1.1986, S.86-87; Ralph de Gubernatis, "Et Pina Bausch créa des monstres", NO, 20.6.1986, S.104 ; "Festival du cinéma des femmes", $N O, 26.9 .1986$; "Bertelsmann: deux fois Hachette", $N O, 3.10 .1986$, S.56.

124 Michel Tournier, "Livres. En Prusse, c'est-à-dire nul part", NO, 26.9.1986, S.102-103; "Expositions: Berlin, l'or des déchirures", NO, 22.3.1986; Michel Meyer, "Les héritiers de Goethe et d'Auschwitz", Flammarion, 1986, NO, 15.3.1986, S.136.

125 Alain de Penanster, "Auschwitz, l'affaire du carmel", EX, 2.5.1986, S.46-47.

126 Jean Baptiste Michel, "Souviens-toi, Berlin, Livres: Josef sauvé par ses frères", EX, 19.9.1986, S.130.

127 Dossier 39-45: "Le secret des archives", EX, 10.10.1986, S. 51-57.

128 Nerin E.Gun, "Document. Et Staline dit oui à Hitler", NO, 15.8.1986, S. 14-15. ders., "Document. Hitler-Staline: partageons-nous le monde", NO, 22.8.1986, S.14-18. 
Vernichtungslagern ${ }^{129}$ und wendet sich mit Nachdruck gegen die Behauptung des Historikers Emil Nolte, „Hitler habe sich nur verteidigt“"130.

1987

In beiden Wochenzeitungen ist 1987 das Interesse an Deutschland doppelt so groß wie 1986. Achtundneunzig Artikel erscheinen, dreiundsechzig in L'Express, fünfunddreißig in Le Nouvel Observateur. Die wichtigsten Themen sind die Aufwertung der DM gegenüber dem französischen Franc sowie die Bundestagswahlen im Januar des Jahres. Hinzu kommt der Prozess gegen den Chef der Gestapo von Lyon, Klaus Barbie.

Am 12. Januar 1987 wird die DM um drei Prozent gegenüber dem Franc aufgewertet, nachdem Bonn die Aufwertung zunächst ablehnte mit der Begründung, es handle sich um eine Schwäche der französischen Währung, auf die Paris zu antworten habe. Die Aufwertung der DM und die Bundestagswahlen vom 25. Januar nimmt $L^{\prime}$ Express zum Anlass für ein Dossier am 23. Januar mit dem Titel Allemagne. Envol d'un supergrand (Deutschland. Höhenflug einer Großmacht). Es möchte in acht Artikeln „alle Wahrheiten über Deutschland“ vermitteln und mit Vorurteilen aufräumen, auch mit jenem, Deutsche hätten keinen Humor. Der „Höhenflug“ betrifft nicht nur die deutsche Währung, sondern auch den Bundeskanzler, den „Schwarzen Riesen aus der Pfalz“, dessen neuer Wahlsieg als sicher gilt. ${ }^{31}$ Jérôme Dumoulin erinnert daran, dass Michel Meyer schon vor drei Jahren die politischen Fähigkeiten Helmut Kohls erkannte, als dieser von vielen noch unterschätzt wurde. Es bestehe kein Grund zur Sorge vor einer Destabilisierung der Bundesrepublik. Zwar gebärde sich die SPD immer neutralistischer und grüner, doch die Chance, bald wieder an die Macht zu kommen, sei gering. Insgesamt habe es Frankreich mit einer ,entspannten und selbstsicheren Bundesrepublik" zu tun, die ihren Wohlstand genießen

\footnotetext{
129 Claude Roy, "Livres. Elles sont vivantes! Quand la photographie se mettait au service de la mort", NO, 7.3.1986, S.94-95.

130 François Schlosser, "Allemagne: Hitler en appel. Quand les Allemands réécrivent l'histoire du nazisme", $N O, 12.12 .1986, \mathrm{~S} .80-81$.

131 Nach der Wahl kommentieren Paul-Jean Franceschini und Elie Marcuse die Regierungsbildung. "R.F.A.: le jeu de patience du chancelier", EX, 6.2.1987, S.37.
} 
will, und wichtig für Europa ist. ${ }^{132}$ Elie Marcuse meint, die SPD werde „radikaler“. Denn der Atlantiker Johannes Rau gerate ins Hintertreffen zugunsten von Oskar Lafontaine und jenen, die „mit den Grünen flirten“, Gerhard Schröder und Volker Hauff. ${ }^{133}$ Die Grünen verdanken ihren zweiten Frühling weniger ihren Spitzenpolitikern Petra Kelly, Otto Schily, Lukas Beckmann und Jutta Ditfurt als vielmehr den ,ökologischen Katastrophen“, mit denen der Rhein und der Wald - „diese der deutschen Seele so teuren Schmuckstücke des Nibelungen“ - zu kämpfen haben. Der Sympathiezuwachs für die Grünen liege auch daran, dass immer mehr Deutsche Misstrauen hegen gegenüber etablierten Parteien und Institutionen. Der Skandal um die „Neue Heimat“ und die „Flick-Affäre“ verunsichern, die Kirchen können keine Orientierung bieten. Mit „Distanz zu Träumern, die es destabilisieren könnten“, werde Deutschland immer grüner. ${ }^{134}$ Zusammen mit Michel Jacques zeigt Elie Marcuse auch, dass die einst armen südlichen Bundesländer Bayern und Baden-Württemberg dank moderner Technologien die Industrieregionen im Nordwesten überrundet haben, da letztere mit Strukturschwierigkeiten kämpfen. Fazit: Die Deutschen ziehen den „Pragmatismus dem Triumphalismus“ vor und fahren dabei nicht schlecht. ${ }^{35}$ Im Laufe des Jahres erörtert L'Express noch einmal die Lage der SPD, die zu „fünfzehn Jahren Fegefeuer", also Opposition, verdammt scheint und auf der Suche nach neuen Wählern immer grüner und pazifistischer werde. ${ }^{136}$ Es interessiert zudem der Popularitätszuwachs der Liberalen ${ }^{137}$ sowie der bayerische Ministerpräsident. Für Elie Marcuse und Paul-Jean Franceschini ist Franz Josef Strau $\beta$ ein ,alternder Teufel“, der immer noch „seine Hörner

\footnotetext{
132 Jérôme Dumoulin, "Allemagne: l'envol d'un supergrand”, EX, 23.1.1987, S.82. Der Artikel bestätigt das zwei Wochen zuvor von Franceschini und Marcuse gezeichnete Bild der Bundesrepublik: "R.F.A.: tranquille comme Kohl", EX, 9.1.1987, S.37.

133 Elie Marcuse, "S.p.d.: le péril gauchiste", EX, 23.1.1987, S.87-88 ; Paul-Jean Franceschini/ Elie Marcuse, Interview mit Oskar Lafontaine, "Les ambitions d'Oskar", ebd., S.88.

134 Elie Marcuse, "Le deuxième printemps des Verts", EX, 23.1.1987,S.90.

135 Michel Jacques/ Elie Marcuse, "Economie: la revanche du Sud", EX, 23.1.1987, S.94-95.

136 Elie Marcuse, "Allemagne. S.p.d.: 15 ans de purgatoire", EX, 10.4.1987, S.30.

137 Paul-Jean Franceschini, "R.F.A.: le mai des libéraux", EX, 22.5.1987, S.36; ders., "R.F.A.: les mécomptes du renversement", EX, 29.5.1987, S.36.
} 
und die Mistgabel“" einsetze. ${ }^{138}$ Im übrigen wirbt Yves Cau um mehr Verständnis für deutsche Positionen. Viele Franzosen machen den Deutschen wegen ihres vermeintlichen „Nationalneutralismus“ den Prozess, ohne ihre Sorgen ernst zu nehmen. ${ }^{139}$ Alain Besançon bemerkt aber solche Tendenzen im gesamten politischen Spektrum, bei Rechten gleichermaßen wie bei Linken und Grünen. Er sieht auch, dass die Auffassungen der Grünen dank der Evangelischen Kirchen in beiden deutschen Staaten immer wichtiger werden. Mit dem „romantischen Geist von 1813 und 1871“ träume man von der Wiedervereinigung. ${ }^{140}$ - Jean-Marc Gonin kritisiert bayerische Vorschläge zur Aidsbekämpfung, Bernard Lecompte kommentiert über die Übersiedlung von viertausend Wolgadeutschen in die Bundesrepublik, Paul-Jean Franceschini informiert über den Tod des Unions-Politikers Uwe Barschel. ${ }^{141}$ Vier Artikel erörtern im September den Bonnbesuch des DDRStaatsratsvorsitzenden Erich Honecker. ${ }^{142}$

In Le Nouvel Observateur kommentiert François Schlosser die Bundestagswahl unter der Überschrift „Der Mann, der den Dollar zittern lässt" und kritisiert erneut die unbeugsame Haltung Helmut Kohls in Zinsfragen. ${ }^{143}$ Der sozialistische Politiker Jean-Pierre Chevènement meint, für die deutsch-französischen Beziehungen sei die Stunde der Wahrheit gekommen, denn beide Länder sind aufeinander angewiesen. Frankreich brauche Deutschland, um ,mit Hilfe des Ecu eine neue finanzielle Identität““ zu entwickeln, die Bundesrepublik benötige Frankreich, um den Zwiespalt auszugleichen, in dem sie sich befindet: einerseits Unterordnung unter die

\footnotetext{
138 Elie Marcuse/ Paul-Jean Franceschini, "R.F.A.: tranquille comme Kohl", EX, 9.1.1987, S.37.

139 Yves Cau, "National-neutralisme?", EX, 22.5.1987, S.37.

140 Alain Besançon, "Ce qui travaille l'Allemagne", EX, 25.9.1987, S.45.

141 Jean-Marc Gonin, "Scènes de chasse en Bavière", EX, 5.6.1987, S.40; Bernard Lecompte, "Les oubliés de la Volga", EX, 7.8.1987, S.23.

Paul-Jean Franceschini, "R.F.A.: grandeur et déchéance d'Uwe Barschel", EX, 16.10.1987, S.38-39.

142 Elie Marcuse, "Bonn: tapis rouge pour Honecker", EX, 4.9.1987, S.34 ; ders., "R.D.A.: les désarrois de l'élève modèle", ebd ., S.36-38; Yves Cau, "L'homme du mur", ebd., S.37; Elie Marcuse, "R.F.A.-R.D.A: à chacun sa vérité", EX., 11.9.1987, S.135-136. 
Vereinigten Staaten, andererseits die Absicht, zwischen Ost und West zu vermitteln. ${ }^{144}$ Jean Daniel und François Schlosser erörtern neue Abrüstungsvorschläge sowie die innerdeutschen Beziehungen anlässlich des Besuchs von Erich Honecker in Bonn. ${ }^{145}$

In L'Express kommentiert Jean-Claude Casanova die deutschfranzösischen Beziehungen mit Blick auf das gespannte Verhältnis zwischen Mark und Franc. Er empfiehlt der französischen Regierung, von Deutschland und der Schweiz zu lernen und den Kapitalverkehr völlig frei zu geben. ${ }^{146}$ Ansonsten interessiert, wie im Vorjahr, der Technologie- und Verteidigungsbereich. Der Beschluss einer deutsch-französischen Brigade wird positiv bewertet. ${ }^{147}$ Das deutsch-französische Herbstmanöver „Kecker Spatz" unter deutschem Oberkommando (17.-24. September 1987) stellt für Jérôme Dumoulin und Elie Marcuse einen Schritt in die richtige Richtung dar; ${ }^{148}$ die technologische Zusammenarbeit erscheint sinnvoll ${ }^{149}$. Es wird betont, dass die Deutschen von Frankreich erwarten, ihre Sicherheit genauso ernst zu nehmen wie die französische. ${ }^{150}$ Als Antwort auf die Kritik von Finanzminister Edouard Balladur an Bonns Zinspolitik, wirbt Jean-Louis Le Marchand um mehr Verständnis für die deutsche Haltung. Paris müsse die eigene Wirtschaft sanieren, da Frankreich trotz erfreulicher Anzeichen immer noch eine höhere Inflationsrate hat als Deutschland, das Außenhandelsdefizit

\footnotetext{
$144 \quad$ Jean-Pierre Chevènement, "France-Allemagne: l'heure de vérité", NO, 30.1.1987, S.52.

145 Jean Daniel, "Des missiles rayés de la carte", EX, 4.9.1987, S.39 ; François Schlosser, "Allemagne : les sirènes d'Erich Honecker", NO, 11.9.1987, S.72.

146 Jean-Claude Casanova, "Le mark et l'Europe", EX, 23.1.1983, S.45.

147 "Europe: la brigade du Rhin", EX, 26.6.1987.

148 Jérôme Dumoulin/ Elie Marcuse, "France-Allemagne: les couacs de Moineau hardi", EX, 2.10.1987, S.25.

149 Françoise Harrois-Monin, "Quand les fusées auront des ailes", EX, 12.6.1987, S.46; Arnaud Rodier, "Quand l'hélicoptère fera avion", ebd., S. 47; Elie Marcuse, "Mitterrand-Kohl: défense d'inquiéter", $E X, 23.10 .1987$, S.32.

150 Jérôme Dumoulin, "Défense. Paris-Bonn: ce que demandent les Allemands", in: Numéro spécial. "Pourquoi nous voulons l' Europe" (Warum wir Europe wollen), EX, 27.2.1987, S.39-40. Siehe auch das Interview von Guillaume Malaurice mit Rudolph von Thadden: "Deux soeurs et dix cousins", ebd., S.51.
} 
fortbesteht und zuwenig investiert wird. ${ }^{151}$ In Le Nouvel Observateur bezeichnet François Schlosser die deutsch-französische Brigade und das Manöver „Kecker Spatz“ als große Herausforderung. ${ }^{152}$

L'Express kommentiert die bundesdeutsche Außenpolitik in vier Artikeln. Paul-Jean Franceschini zeichnet ein freundliches Porträt von Bundesaußenminister Hans-Dietrich Genscher unter der Überschrift „Die Diplomatie des Elefanten“. Er erörtert die Genscher-Doktrin im Nahen Osten am Beispiel der Bonner Bemühungen um die Freilassung der deutschen Geiseln im Libanon: Wirtschaftsbeziehungen mit allen Ländern der Region, strikte Neutralität im Krieg zwischen Iran und Irak, kein Nachgeben gegenüber Erpressern und Fortsetzung des politischen Dialogs. ${ }^{153}$ In Bezug auf das zweite außenpolitische Thema, die Ost-Westbeziehungen, äußert Elie Marcuse Zweifel an der von der Sowjetunion vorgeschlagenen Null-Lösung und den „unsicheren Annäherungen“ zwischen Bonn und Moskau. ${ }^{154} \mathrm{Le}$ Nouvel Observateur thematisiert deutsche Außenpolitik nicht eigens.

Zum Thema Wirtschaft präsentiert L'Express ein vielschichtiges Bild in neun Artikeln. Ein Dossier über die „Eurocitys“ - Städte, die dank eines „Europas der Regionen“ an Bedeutung gewinnen werden - zeichnet ein wirtschaftliches und kulturelles Porträt von Hamburg, Frankfurt und vor allem München. ${ }^{155}$ Michel Jacques und Marie Pierre Gröndahl weisen darauf hin, dass der niedrige Dollarkurs die deutsche Wirtschaft, besonders Volkswagen, belaste, da der Export in Dollar abgewickelt wird. Die Leser werden auch informiert über die steigende Bereitschaft der Deutschen zu

\footnotetext{
151 Jean-Luc Le Marchand, "Economie. Franc-mark: le divorce", EX, 6.11.1987, S.46-48.

152 François Schlosser, "Brigade franco-allemande: le grand défi", NO, 26.6.1987, S.33-34 ; ders., "France-Allemagne: 'Moineau hardi' sur le Danube", NO, 25.9.1987, S.50.

153 Paul-Jean Franceschini, "Genscher: la diplomatie de l'éléphant", EX, 3.4.1987, S.64; ders., "Otages: les silences de Bonn", EX, 13.3.1987, S.31.

154 Elie Marcuse, "Désarmement. Option zéro: rien n'est joué", EX, 3.4.1987, S.36 ; ders., "Moscou-Bonn: incertain sourire", EX, 17.7.1987, S.25.

155 "Dossier Eurocités, "Milan s'entend comme larron en foire avec Munich", EX, 18.12.1987, S.84.
} 
Kreditaufnahmen ${ }^{156}$ sowie Auseinandersetzungen zwischen den Sportwarenherstellern Adidas und Puma. ${ }^{157}$ Le Nouvel Observateur veröffentlicht kein Dossier zur Aufwertung der deutschen Währung, kommentiert das Ereignis aber unter der Überschrift „,Der Gefreite Müller wird befördert"“. Georges Valence kritisiert die Weigerung der Bundesbank, die Leitzinsen zu senken und der DM-Aufwertung nur zögernd zugestimmt zu haben. Nicht namentlich genannten Mitarbeitern des französischen Finanzministeriums zufolge „leben die deutschen Führungspersönlichkeiten weitab der großen Metropolen und leiden an jenem Provinzialismus, den Madame de Staël zu Beginn des neunzehnten Jahrhunderts so treffend beschrieben habe“.158 Zum Thema Gesellschaft beschreibt Chantal Rudder in Le Nouvel Observateur die multikulturelle Stadt Frankfurt am Main, in der jeder dritte Einwohner Ausländer ist. ${ }^{159}$ Sechs Artikel feiern den siebenhundertfünfzigsten Geburtstag von Berlin unter der Überschrift „,750 Kerzen für die deutsche Frage“. ${ }^{160}$ Auch der neue BMW 750, das „Auto der Generaldirektoren“, findet Beachtung. ${ }^{161}$

$\mathrm{Zu}$ Kultur veröffentlicht L'Express sieben, Le Nouvel Observateur zehn Artikel. L'Express berichtet über die Pariser Premiere von Wim Wenders Film „Der Himmel über Berlin“ (übersetzt mit „Les ailes du désir“, Die Flügel der Begierde) und den Hauptdarsteller Peter Falk. Ein Kommentar zu den

\footnotetext{
156 Michel Jacques, "Economie. L'Allemagne sous pression", EX, 4.12.1987, S.54-55; MariePierre Gröndahl, "Economie. Volkswagen perd au change", EX, 20.3.1987, S.38-40 ; Michel Jacques, "R.F.A.: prêts, repartez!", EX, 3.7.1987, S.44.

157 Jean-Marc Gonin, "Economie. Puma-Adidas: balle de match?", EX, 21.8.1987, S.32.

158 Georges Valence, "Le 'Caporal Muller' prend du galon", NO, 16.1.1987, S.39-40. Diese Ausgabe erörtert auch, wie Deutsche Frankreich und Japaner Deutschland sehen. "La France vue d'Allemagne", ebd., S.41, "L'Allemagne vue du Japon", ebd , S.44.

159 Chantal Rudder, "Kanaks, Kartofels, beurs and Co ", NO, 27.3.1987, S.80-81.

160 Daniel Rondeau, "Berlin: 750 bougies pour la question allemande", NO, 15.5.1987, S.110 ; "Allemagne: année zéro, souvenirs du lieutenant Jean Rauch", ebd., S.112; Rudolph von Thadden, "Histoire des tribus allemandes, La chronique des gares berlinoises", ebd., S.114 ; Annie Cohen-Solal, "Cabarets", ebd., S.120; Cathérine David, "Gens de Berlin. Friedrich Gorenstein: un hôte de passage", ebd .,S.117; Daniel Rondeau, "Gens de Berlin. MarieLouise von Plessen: la comtesse des musées sentimentaux", ebd ., S.119. 
Berliner Filmfestspielen verdeutlicht das Interesse an russischen Produktionen. ${ }^{162}$ Buchbesprechungen ergänzen die Berichterstattung. Werke von Jürgen Habermas, Max Weber, Christa Wolf und Friedrich Nietzsche werden vorgestellt. ${ }^{163}$ Im Nouvel Observateur erörtert Fritz Raddatz Kommunikationsschwierigkeiten zwischen deutschen Schriftstellern und den Medien. Daniel Rondeau interviewt den Verleger Ernst Rowohlt und ein Bericht über den „Berliner Tag“ im französischen Fernsehsender La Sept rundet das Bild ab. ${ }^{164}$ Pina Bausch wird anlässlich ihres Pariser Gastspiels gewürdigt, Pierre Ajame interviewt Hans Jürgen Syberberg. ${ }^{165}$ Le Nouvel Observateur veröffentlicht ein Interview von Didier Eribon und Ruth Valentini mit Jürgen Habermas über die Theorie des kommunikativen Handels ${ }^{166}$, Jacques Derrida kommentiert Victor Farias Buch Heidegger et le nazisme. ${ }^{167}$ Norbert Elias und Walter Benjamin werden ebenfalls thematisiert, desgleichen das in Frankreich entdeckte Bild des Künstlers von Ernst Kris

162 François Forestier, "Cinéma. Peter Falk: un ange passe à Berlin", EX, 18.9.1987, S.121125 ; Peter Kral, "Moscou sous l'œil de Berlin", EX, 27.3.1987, S.113.

163 Raymond Boudon/ Jean Marc Fery/ Jean-Louis Schlegel: "Habermas, tout communique", Théorie de l'agir communicationnel, Fayard 1987, EX, 5.6.1987, S.156-158; Alain Renault, "Livres: Weber. Déjà le philosophe allemand disséquait la raison moderne" (Philippe Raynaud, Max Weber et les dilemmes de la raison moderne PUF, 1987), EX 11.9.1987, S.114; Paul-Jean Franceschini, "Christa Wolf: le long retour", EX, 16.10.1987, S.166; Alain Renault, "Quoi de neuf? Nietzsche." (Aurore, Hachette, 1987), EX, 23.10.1987, S.168; Yves Cau, "Les cartons de Berlin", EX, 27.3.1987, S.35.

164 Fritz Raddatz "Hambourg: tout par la radio", NO, 20.3.1987; Daniel Rondeau, "Berlin: les années Rowohlt. Un entretien avec le monstre sacré de l'édition allemande", NO, 5.6.1987, S.24-25: "Télévision: un jour entier à Berlin", NO, 5.6.1987, S.22.

165 Ralph de Gubernatis, "La gloire de Pina Bausch", NO, 10.4.1987, S.92-94 ; Pierre Ajame, "Kleist, mon compatriote", Interview mit Hans Jürgen Syberberg und Bericht über Edith Clever, NO, 6.11.1987, S.160-162.

166 Didier Eribon/Ruth Valentini, Interview mit Jürgen Habermas, "Triomphe de la philosophie", NO, 20.3.1987, S.118-119.

167 Didier Eribon, Interview mit Jacques Derrida, "Heidegger, l'enfer des philosophes", NO, 6.11.1987, S.170-174. 
und Otto Kurz. ${ }^{168}$ Wie L'Express bespricht Le Nouvel Observateur die Neuauflage der französischen Übersetzung von Nietzsches Morgenröthe. ${ }^{169}$

Geschichte wird thematisiert anlässlich des Barbie-Prozesses in Lyon. Dies führt jedoch in beiden Wochenzeitungen zu keiner Verschlechterung des Deutschlandbildes, gibt aber Anlass zu mehr Auseinandersetzung mit der Kollaboration. ${ }^{170}$ Zum Nationalsozialismus veröffentlicht Le Nouvel Observateur zudem ein Interview mit Claude Lanzmann über seinen Film $S_{\text {Shoh }}{ }^{171}$ und berichtet über Primo Levi und Anne Frank. Auch das Buch von Walter Laqueur Auschwitz, you say Auschwitz? Wird rezensiert, das den amerikanischen Präsidenten Roosevelt der unterlassenen Hilfeleistung in Bezug auf die Shoah anklagt. ${ }^{172}$ L'Express thematisiert den Nationalsozialismus in einem Artikel über Hitlers Stellvertreter Rudolph Hess, den „Letzten der Verdammten“, ${ }^{173}$ in einer Besprechung des Theaterstücks „Wannsee, Vorzimmer des Todes“"174 und einer Würdigung von Anne Franks Familie. ${ }^{175}$ Alain Finkielkraut präsentiert das Buch von André Glucksmann Descartes c'est la France unter dem Titel „Descartes, Auschwitz et nous“. ${ }^{176} \mathrm{Im}$ Rahmen des Dossiers „Allemagne. Envol d'un

168 Didier Eribon, "Livres. Le vieil homme et la mort", NO, 27.2.1987, S.78-79; ders., "L'art de s'égarer", $N O$, 6.3.1987, S.96 ; Pascal Quignard, "Quelle mouche pique la création?", $N O$, 13.11.1987.

169 Mona Ozouf, "Les vérités alternatives. Un Nietzsche inattendu, à redécouvrir", $N O$, 25.9.1987, S.120.

170 Titelgeschichte des Nouvel Observateur vom 17.4.1987: „Les Collabos“. Artikel in L'Express zu dieser Thematik: Pierre Accoce/ Gérard Chauvy, "Enquête. Barbie: le renfort de la "car-lingue", EX, 12.6.1987, S.38-39; Pierre Accoce, "Affaire Barbie: l'heure du duel", $E X, 26.6 .1987$, S.32-33 ; ders., "France. Barbie: l'histoire a jugé", EX, 10.7.1985, S.26.

171 Cathérine David, Enfin "Shoah"!, Interview mit Claude Lanzmann, NO, 26.6.1987, S.74 ; dies., "La mort au détail. Auschwitz a rattrapé l'écrivain Primo Lévi", NO, 25.9.1987, S.115 ; dies., "La passion d'Anne Frank", NO, 17.4.1987, S.110.

172 Walter Laqueur, L'Abandon des Juifs, Flammarion, NO, 10.4.1987,S. 107.

173 Pierre Accoce, "Hess, le dernier des damnés", EX, 21.8.1987, S.24-26.

174 "Spectacle. Wannsee, antichambre de la mort", EX, 4.12.1987, S.124.

175 Anne Pons, "Livres. Souvenir de la maison des Frank," Calman-Levy, EX, 17.4.1987, S.120121. 
supergrand" (Höhenflug einer Supermacht) nimmt Paul-Jean Franceschini Stellung zum deutschen Historikerstreit. Noltes These, Hitler habe sich in erster Linie gewehrt, dürfe nicht unwidersprochen bleiben. Deutschland könne keinen Schlussstrich unter seine Vergangenheit ziehen, indem es auf Verbrechen anderer verweist. ${ }^{177}$

\section{8: Dossiers zum 25. Jahrestag des Elysée-Vertrages}

Wurden dem zwanzigsten Jahrestag des Elysée-Vertrages keine Dossiers gewidmet, so hat sich die Situation fünf Jahre später geändert. Zum fünfundzwanzigsten

Jubiläum erscheinen in beiden Wochenzeitungen umfangreiche Dossiers über das Nachbarland. Jenes von L'Express hat den Titel „FranceAllemagne: Le drôle de couple“ (Frankreich- Deutschland: das seltsame Paar), Le Nouvel Observateur wählt die Überschrift „Spécial Allemagne. Français, si vous saviez" (Franzosen, wenn ihr wüsstet).

Das Dossier von L'Express ${ }^{178}$ beleuchtet das ,seltsame Paar“ aus verschiedenen Perspektiven, auch in der Privatsphäre. Wie kommen deutschfranzösische Ehepaare miteinander aus? In der Politik betont Paul-Jean Franceschini die „Selbstverständlichkeit“ der deutsch-französischen Beziehungen, obgleich ,nicht alle Zweifel ausgeräumt“ seien. Ein „,seltsames Klima der Hassliebe“ charakterisiere diese „,beispielhafte Ehe“. Der Journalist zeichnet auch ein Porträt des Ministerpräsidenten von Baden-Württemberg, Lothar Späth, den er den „Ersten König der Schwaben“ nennt. Jean-Marc Gonin und Elie Marcuse bedauern, dass trotz des Flugzeugs Airbus und der Rakete Ariane das deutsche Interesse an bilateralen Gemeinschaftsprojekten

177 Paul-Jean Franceschini, "Nazisme: peut-on tourner la page?", EX, 23.1.1987, S.86.

178 "France-Allemagne: le drôle de couple", EX, 15.1.1988 ; Paul-Jean Franceschini, "FranceAllemagne: le drôle de couple", S.65-66; Jean-Marc Gonin, "Grands projets, petits pas", ebd, S.66-67; Elie Marcuse, "Défense: Paris va de l'avant", ebd., S.68; Brigitte Sauzay, "Jeunes: chacun son rêve", ebd., S.70; Michel Candellier, "Tous faibles en thème", ebd.,S.72 73; JeanMarc Gonin, Interview mit Klaus von Bismarck, "Goethe sans peine", S.73; Klaus-Peter Schmid, "Presse: deux styles la Une", ebd., S.74; Bernard Lortholary, "Disciplinés, mais tellement romantiques", ebd., S.75; Klaus Gerth, "Mon village à l'heure de 1992", ebd., S.76; Guillaume Malaurie, "Ich liebe dich, moi non plus", ebd., S.77 ; Paul-Jean Franceschini, "Lothaire $1^{\mathrm{er}}$ roi des Suabes", ebd.,S.78-79. 
gering bleibe. Es kommen auch drei in den deutsch-französischen Beziehungen aktive Deutsche zu Wort: Klaus von Bismarck, Präsident der Goethe-Institute, Klaus-Peter Schmid, Frankreich-Korrespondent des Nachrichtenmagazins Der Spiegel und Klaus Gerth, Deutschlehrer aus der Gegend von Lille. Klaus von Bismarck vergleicht die Selbstdarstellung der deutschen Goethe-Institute und der Centres Culturels Français, Klaus-Peter Schmid zeigt Unterschiede zwischen deutschem und französischem Journalismus auf: Während in Frankreich die Suche nach stilistischer Eleganz dominiert, bevorzugt die deutsche Presse einen Enthüllungsjournalismus nach amerikanischem Vorbild. Die Distanz zwischen Medien und Politik ist in Franreich größer als in Deutschland. In Bonn suchen die Politiker die Nähe der Journalisten, was letzteren zwar die Arbeit erleichtert, doch auch ihre Kritikbereitschaft beeinträchtigen kann. Die Dolmetscherin bei deutschfranzösischen Gipfeltreffen Brigitte Sauzay analysiert Unterschiede zwischen jungen Deutschen und Franzosen. In Frankreich heißen die Referenzwerte Pascal und der Weltraum, in Deutschland Luther und die Umwelt. Verschiedene Traditionen prägen nach wie vor das Verhalten in beiden Ländern. Brigitte Sauzay zufolge findet das deutsche Organisationstalent eine neue Ausdrucksform in der Organisation von Menschenketten gegen die Rüstung. Die Tradition des Protestantismus erlaube es den Deutschen zudem, ihre Überzeugungen mit Nachdruck zu vertreten. In Frankreich herrscht dagegen der Geist von Descartes und der Zweifel - bis hin zur Anerkennung des Absurden. ${ }^{179}$ Der Übersetzer Bernard Lortholary beklagt, dass die „französische Deutschland-Debatte“ diesen Namen nicht wirklich verdiene, da fundamentale Wandlungen nicht wahrgenommen werden. Die Franzosen verstehen nicht, dass das Land von Clausewitz, Bismarck und Hitler pazifistisch geworden und das Ziel der Friedensbewegung wirklich der Frieden ist. Der Mythos verhindere die Wahrnehmung von Gemeinsamkeiten zwischen beiden Ländern sowie von gemeinsamen Problemen. In Kultur und Politik wüssten die Franzosen heute weniger über Deutschland als zu Zeiten von Madame de Staël. ${ }^{180}$

\footnotetext{
179 Brigitte Sauzay, "Jeunes: à chacun son rêve", $E X, 15.1 .1988$, S.70.

180 Bernard Lortholary, "Disciplinés, mais tellement romantiques", $E X, 15.1 .1988$, S.75.
} 
Das Dossier von Le Nouvel Observateur ${ }^{181}$ analysiert folgende Themenbereiche: deutsch-französische Beziehungen, europäische Verteidigung, innerdeutsche Wirtschaftsbeziehungen, Ökologie, Niedergang der politischen Rechten, Suche nach einer neuen nationalen Identität. Es ermöglicht sechs Deutschen, ihre Sicht der Bundesrepublik vorzustellen. Es handelt sich um drei Politiker der SPD, Freimut Duve, Peter Glotz und die Europa-Abgeordnete Beatrice Weber sowie den Präsidenten des Deutschen Industrie- und Handelstages Franz Schoser, den Feuilletonchef der Wochenzeitung Die Zeit Fritz Raddatz sowie Walter Schütze vom Pariser „Institut Français des Relations Internationales“. Die Interviews mit diesen Persönlichkeiten kommentierend, betonen Josette Alia und Ruth Valentini, dass die gegenseitige Unkenntnis zwischen Deutschen und Franzosen groß bleibe. Trotz offizieller Bekenntnisse zu mehr Gemeinsamkeit, sieht man in Paris und Bonn die Zukunft verschieden. Frankreich entdeckt die Notwendigkeit einer europäischen Verteidigung, doch die Deutschen haben daran „kein Interesse“. Ähnliches gelte auch in Hinblick auf das für 1992 vorgesehene „Europa ohne Grenzen“. Während Frankreich sich hierfür zu begeistern beginnt, sehen die deutschen Industriellen ihre Zukunft bereits auf dem Weltmarkt. Die meisten Missverständnisse sind aber nicht politischer, sondern kultureller Art. Noch immer verstellen Stereotypen die Wahrnehmung des Partnerlandes. ${ }^{182}$

Fazit

181 "Spécial Allemagne: Français, si vous saviez", NO, 15.1.1988, S.40-46; Josette Alia/ Ruth Valentini, "Allemagne: Français, si vous saviez", ebd., S.40-41; Josette Alia, "Défense. France Allemagne: le grand quiproquo" (Interview mit Walter Schütze), ebd., 42-43; dies., Interview mit Franz Schoser, Präsident des Deutschen Industrie- und Handelstags: "Economie. Entre Allemands, fini la langue de bois", ebd. S.46; Béatrice Weber, "Ecologie. Ce que les Français ne comprennent pas", ebd., S.43 ; Ruth Valentini/ François Schlosser, Interview mit Fritz Raddatz, ebd., S. 44-45; Freimut Duve, "Nous sommes sortis de l'impasse nationaliste", ebd., S. 44; Peter Glotz, "Opinion. Le déclin de la droite", ebd., S. 45. Henri Louis de la Grange stellt das Buch von Françoise Giroud vor: "Alma Mahler, la fiancée du vent", ebd., S.80. Am 8.Januar 1988 bespricht Alain Riou Werner Herzogs Film "Cobra Verde. NO, 8.1.1988, S.84. 
Wenn am Ende des zwanzigsten Jahrhunderts Franzosen weniger über Deutschland wissen als zu Beginn des neunzehnten Jahrhunderts (wie Bernard Lortholary in L'Express schreibt) und Stereotypen noch immer die Sicht trüben (Le Nouvel Observateur), so ist dies mit Sicherheit nicht allein der Presse anzulasten. Vielmehr sollte diese bedauerliche Bestandsaufnahme als Appell an alle Mittler im deutsch-französischen Dialog gelten, mehr und präziser über Deutschland zu informieren. Erfreulicherweise hat sich das Deutschlandbild in Le Nouvel Observateur aber sehr gebessert im Vergleich $\mathrm{zu}$ den siebziger Jahren. Gleichwohl ist zu kritisieren, dass beide Wochenzeitungen mehrere Themenbereiche nicht kommentieren, die in den achtziger Jahren die öffentliche Meinung in der Bundesrepublik bewegten und für Schlagzeilen sorgten. Zum Beispiel Entwicklungen unter dem Stichwort „Alternatives Wirtschaften“ die an Bedeutung gewannen.183 Oder die Auseinandersetzungen um die atomare Wiederaufbereitungsanlage von Wackersdorf im Jahr 1986. Dieses Ausblenden wichtiger Entwicklungen und Ereignisse ist wohl in erster Linie darauf zurück zu führen, dass Zeitungen prinzipiell aus der Masse an Informationen jene heraus suchen, die ihnen für ihre Leser am interessantesten erscheinen. Und diese Auswahl wird mitbestimmt von der Frage, inwieweit ein Ereignis im Ausland für das eigene Land wichtig ist oder werden kann. Obige Themen erschienen also aus französischer Sicht wenig relevant. Man wollte aber vielleicht auch nicht dazu beitragen, dass durch Berichte über Wackersdorf eine Diskussion über die Kernkraft in Frankreich angeregt werden könnte.

Der Vergleich des Deutschlandbildes von L'Express und Le Nouvel Observateur zeigt viele Gemeinsamkeiten und eine Reihe von Unterschieden. Zudem kommen in jeder der beiden Wochenzeitungen unterschiedliche Meinungen zum Ausdruck. „Müssen wir Angst vor den Deutschen haben?“ betitelt Le Nouvel Observateur 1984 sein Deutschland-Dossier und verneint die Frage. Gleichwohl dokumentieren beide Presseorgane eine gewisse Besorgnis in Bezug auf das Nachbarland. Sie befürchten, Frankreich könne in Zukunft für die Bundesrepublik weniger wichtig sein. Das „Schielen nach anderen Bräuten“, besonders in Richtung Osten, wird argwöhnisch beobachtet.

183 Cf. "Kollektiver Kommerz", Wirtschaftswoche $\mathrm{n}^{\circ}$ 40, 27.9.1985. 
Immer wieder stellen sich die Fragen: Ist Bonn für Paris ein verlässlicher Partner? Engagiert sich die Bundesrepublik genügend bei der Konstruktion Europas? Werden die innerdeutschen Beziehungen zur Konkurrenz für die deutsch-französischen Beziehungen? Respektiert Deutschland seine Verpflichtungen im Rahmen der NATO? Dabei erklären allerdings weder L'Express noch Le Nouvel Observateur ihren Lesern, dass der "Spagat“ zwischen den deutsch-französischen und den deutsch-amerikanischen Beziehungen bereits seit den sechziger Jahren besteht und nicht erst die Politik der achtziger Jahre bestimmt.

Die Deutschlandberichterstattung von L'Express und Le Nouvel Observateur beschränkt sich nicht auf die Bundesrepublik. Sie informiert auch über Entwicklungen der DDR und nimmt die „Identitätskrise der geteilten Nation“ ernst. In Bezug auf die Friedensbewegung und die damit verbundene Rolle der Grünen in der politischen Landschaft herrscht Befremden und weitgehendes Unverständnis. Die sogenannten „Ökopazifisten“ bereiten Sorge. Um so mehr als beide Presseorgane die Notwendigkeit einer europäischen Verteidigung betonen und gemeinsame Technologieprojekte befürworten, auch im militärischen Bereich. Im gesamten Untersuchungszeitraum wird bedauert, dass Bonn sich hier zu wenig engagiere. Doch es wird auch erklärt, Frankreich müsse mehr Verständnis für die deutsche Haltung aufbringen und sich stärker für Deutschlands Sicherheit einsetzen. Mit Interesse und Misstrauen wird der Wandlungsprozess der SPD betrachtet, an der Politik der Bundesregierung irritieren „Zweideutigkeiten“. Gleichwohl zeichnet L'Express ein positives Bild des Kanzlers: Helmut Kohl gilt als Garant gegen neutralistische Strömungen und als Befürworter deutschfranzösischer und europäischer Technologieprojekte. In Währungs- und Wirtschaftsfragen bedauern beide Presseorgane einen gewissen deutschen Druck auf Frankreich. L'Express betont die Leistungsfähigkeit der deutschen Wirtschaft und leitet daraus Empfehlungen für Frankreich ab, Le Nouvel Observateur interessiert sich stärker für soziale Konsequenzen der Modernisierung. Beide Wochenzeitungen registrieren einen Mentalitätswandel: 1983 und 1984 herrschte ein „Klima der Angst“, die Möglichkeit eines Dritten Weltkriegs und Arbeitslosigkeit beunruhigten. Doch Mitte der achtziger Jahre veränderte sich die Stimmung zugunsten einer 
materialistischen und hedonistischen Lebenseinstellung. Zugleich entwickelte sich ein ,grünes Kulturideal“".

Die meisten Artikel gehören dem Kulturbereich an - wir finden achtundvierzig in Le Nouvel Observateur und fünfundvierzig in L'Express. Dabei handelt es sich teilweise um Informationen über das kulturelle Leben der Bundesrepublik, doch meistens, neben Buchbesprechungen, um deutsche Kultur, die sich in Frankreich vorstellt, auf der Bühne, im Film und in Ausstellungen. Besondere Sympathie genießt Pina Bausch, die den Beinamen „Fee von Wuppertal“" erhält. Am wenigsten interessiert deutsche Außenpolitik, vor allem wenn sie den europäischen und nordatlantischen Rahmen sowie die Ost-West-Beziehungen überschreitet: zehn Artikel in L'Express, fünf in Le Nouvel Observateur. Die hohe Frequenz der nationalsozialistischen Vergangenheit (einunddreißig Artikel in L'Express, zweiunddreißig in Le Nouvel Observateur) wird vor allem erreicht durch Buchbesprechungen und Kommentare zu folgenden Ereignissen: Entdeckung des Grabes von Josef Mengele, Besuch Helmut Kohls und Ronald Reagans auf dem Soldatenfriedhof von Bitburg, Prozess gegen Klaus Barbie, Claude Lanzmanns Film „Shoah“, Historikerstreit. Beide Presseorgane reagieren sensibel, wenn versucht wird, die Verantwortung der Deutschen für die Verbrechen des Nationalsozialismus zu relativieren. Andere Aspekte der deutschen Geschichte interessieren kaum: Luther wird zur Erklärung aktueller Entwicklungen bemüht und Le Nouvel Observateur informiert zudem über die Emigration französischer Hugenotten nach Preußen als Folge der Aufhebung des Edikts von Nantes.

Die Qualität der Deutschlandberichterstattung von L'Express und Le Nouvel Observateur wird dadurch bestätigt, dass deutsche Persönlichkeiten aus Politik, Wirtschaft und Kultur zu Wort kommen, in Interviews und Kommentaren. Die unterschiedliche Schwerpunktsetzung erkennt der Leser auch daran, dass die Namen von dreiunddreißig Deutschen in Artikelüberschriften erscheinen, aber nur sechzehn davon in beiden Zeitungen. Es handelt sich um sechs Politiker - Willy Brandt, Erich Honecker, Helmut Kohl, Oskar Lafontaine, Franz Josef Strauß, Hans Jochen Vogel - und neun Vertreter des Kulturlebens: Pina Bausch, Günter Grass, Michael Grüber, 
Jürgen Habermas, Heiner Müller, Peter Schneider, Peter Stein, Hans Jürgen Syberberg und Wim Wenders.

Abschließend kann man sagen: L'Express und Le Nouvel Observateur vermitteln in den achtziger Jahren, zwischen 1983 und 1988, ein vielschichtiges und nuancenreiches Bild des Nachbarlandes, das zum Teil kritisch ist. Sie ergänzen sich dabei in ihrer Schwerpunktsetzung. Jean Daniel schreibt 1983, dass Frankreich und Deutschland ,im gleichen Boot“ sitzen und diese Einsicht spiegelt sich im gesamten Untersuchungszeitraum im Deutschlandbild beider Wochenzeitungen.

\section{Literaturverzeichnis}

GROSSER, Alfred Grosser, "Das Wenige wahren", Frankfurter Allgemeine Zeitung, 1.10.1986

HÖRLING, Hans, „L'image de la République fédérale allemande (RFA) dans la presse hebdomadaire française", in: L'Allemagne vue par la presse française. Cahiers de l'Association pour une lecture critique de la presse, Paris, 23.10.1978, S.14-23.

MENUDIER, Henri (Hrsg.), La presse et les relations franco- allemandes. Facteur de compréhension ou de mésentente?, Paris, Goethe-Institut, 1978.

MENUDIER, Henri, Das Deutschland der Franzosen in den 70er Jahren, Europa Union Verlag, Bonn 1981

PINTO, Louis, L'intelligence en marche : Le Nouvel Observateur, Editions Métaili, Paris 1984

REVEL, Jean-François, L'Express. L'aventure du vrai, Albin Michel, Paris 1979

SCHOBER, Angelika, „Bayern und München in den französischen Wochenzeitungen L'Express und Le Nouvel Observateur 1983-1988“ „La Bavière et Munich dans les hebdomadaires français L'Express et Le Nouvel Observateur 1983-1988“, in: Pierre Albert, Ursula Koch, Detlef Schröder (Hrsg.), Deutsch-französische Medienbilder. Journalisten und Forscher im Gespräch/ Images médiatiques franco-allemandes. Un dialogue entre journalistes et chercheurs, Verlag Reinhard Fischer, München 1993, S.171-186. 